\title{
Integration of reward with cost anticipation during performance monitoring revealed by ERPs and EEG spectral perturbations
}

\author{
Davide Gheza ${ }^{1}$, Rudi De Raedt ${ }^{2}$, Chris Baeken ${ }^{3-5}$ \& Gilles Pourtois ${ }^{1}$
}

(1) Cognitive and Affective Psychophysiology Laboratory, Department of Experimental Clinical \& Health Psychology, Ghent University, Ghent, Belgium

(2) Psychopathology and affective neuroscience Laboratory, Department of Experimental Clinical \& Health Psychology, Ghent University, Ghent, Belgium

(3) Department of Psychiatry and Medical Psychology, Ghent University, Ghent, Belgium

(4) Department of Psychiatry, University Hospital (UZBrussel), Brussels, Belgium

(5) Ghent Experimental Psychiatry (GHEP) lab, Ghent University, Ghent, Belgium

Corresponding author: Gilles Pourtois

Department of Experimental Clinical and Health Psychology, Ghent University

Henri Dunantlaan 2

9000 Ghent, Belgium

Phone: +3292649144

E-mail: gilles.pourtois@ugent.be 


\begin{abstract}
Effort expenditure has an aversive connotation and it can lower hedonic feelings. In this study, we explored the electrophysiological correlates of the complex interplay of reward processing with cost anticipation. To this aim, healthy adult participants performed a gambling task where the outcome (monetary reward vs. no-reward) and its expectancy were manipulated on a trial by trial basis while 64-channel EEG was recorded. Crucially, on some trials, the no-reward outcome could be transformed to a rewarding one, pending effort expenditure by means of an orthogonal dot clicking task, enabling us to compare at the electrophysiological level reward processing when cost was anticipated or not. We extracted and compared different markers of reward processing at the feedback level using both classical ERPs and EEG spectral perturbations in specific bands (theta, delta and beta-gamma). At the behavioral level, participants reported enhanced pleasure and relief when the outcome was rewarding but effort expenditure could be avoided, relative to a control condition where the outcome was rewarding but no extra effort was anticipated. In this condition, EEG results showed a larger Reward Positivity ERP component and increased power in the Delta and Beta-gamma bands. By comparison, cost anticipation did not influence the processing of the no-reward outcome at the FRN and frontal midline theta levels. All together, these neurophysiological results suggest that effort avoidance is associated with increased reward processing.
\end{abstract}

Keywords: reward, effort, opportunity cost, performance monitoring, Reward Positivity, ACC 


\section{Introduction}

Humans tend to obey to a principle of economy ("law of less work"; Hull, 1943). This principle applies to both physical and cognitive effort (Apps et al., 2015; Kool et al., 2010), whereby rewards are devalued by the cost required to obtain them (Charnov, 1976; Salamone et al., 2007). An increasing interest on motivational and emotional processes underlying decision making, where the integration of effort with reward occupies a central place, has been witnessed recently in a wide range of disciplines, spanning from neuroeconomics (Westbrook and Braver, 2015) to psychopharmacology (Salamone et al., 2012) and neuroscience (Apps et al., 2015; Chong et al., 2017; Ma et al., 2014; Vassena et al., 2014). These valuable efforts have substantially advanced our understanding of how motivation shapes decision making, especially from a computational perspective that provides mechanistic accounts to explain brain mechanisms responsible for value processing and effort deployment (Holroyd and McClure, 2015; Kurzban et al., 2013; Vassena et al., 2017; Verguts et al., 2015). In this literature, the dorsomedial and dorsolateral prefrontal cortex are often considered as domain-general brain regions involved in reward (d)evaluation when encountering either cognitive or physical effort (Chong et al., 2017). In particular, the anterior cingulate cortex (ACC) and the striatum are thought to signal effort anticipation (Kurniawan et al., 2013, 2010), and to process the expectation of both reward and cognitive effort (Vassena et al., 2014). At the electrophysiology level, neural activity arising from the ACC has traditionally been related to specific performance monitoring (PM) or cognitive control (CC) ERP components, such as the ERN (Error related negativity) and FRN (Feedback related negativity; see Holroyd and Coles, 2002). PM is a complex ability relying on different and interconnected mental processes, including an early evaluative component, in case errors or mismatches are detected and need to be rapidly processed to foster goal-adaptive behavior. At the electrophysiological level, this early evaluative component has been related to specific EEG markers, elicited both in the time and time-frequency domains (Ullsperger et al., 2014b). 
In the time-domain, the FRN component is usually defined as a negative ERP deflection peaking at around $250 \mathrm{~ms}$ at channels FZ or FCZ after evaluative feedback (FB) onset. FB is characterized as evaluative since it provides information about performance outcome in the present case. FRN's amplitude is enhanced after negative vs. positive, and unexpected vs. expected FB, thus providing an electrophysiological marker of PM sensitive to both outcome expectation and valence information (Holroyd and Coles, 2002; Ullsperger et al., 2014a; Walsh and Anderson, 2012). Traditionally, the negative deflection (i.e. N200) giving rise to the FRN has been linked to a phasic and signed reward prediction error (RPE) signal (Holroyd and Coles, 2002). More specifically, it conveys the direction of the deviation between the actual and the expected outcome. This phasic signal is thought to be generated first in deep dopamigeric structures (midbrain), before it is relayed to the medial prefrontal cortex, including the ACC which is thought to provide the main intracranial generator of the FRN. Whereas dopamine has usually been put forward as the main neurotransmitter accounting for RPE in the context of reinforcement learning and PM, more recently, other neurotransmitter systems have also been considered in this process. These include norepinephrine (Riba et al., 2005) and the involvement of the locus coeruleus in decision-making (Aston-Jones and Cohen, 2005), GABA (reducing the amplitude of the ERN; De Bruijn et al., 2004), but also serotonin and adenosine (for a review see Jocham and Ullsperger, 2009). The cognitive processes giving rise to PM, its neural underpinning as well as its electrophysiological signature, are still debated in the current literature. For instance, with regard to the FRN, the ERP amplitude difference between negative and positive FB has been interpreted as a positivity associated with better than expected outcome (Eppinger et al., 2008; Holroyd et al., 2008; Holroyd and Umemoto, 2016; Sambrook and Goslin, 2014). Accumulating evidence indicates that such an outcome-dependent amplitude difference may be driven by sensitivity to rewarding rather than non-rewarding events (Arbel et al., 2013; Baker and Holroyd, 2011; Foti et al., 2011; Potts et al., 2006; Sambrook and Goslin, 2014; Weinberg et al., 2014), leading thereby some authors to name this ERP component Reward Positivity (RewP; for a review, see Proudfit, 2015), as opposed to FRN. Although sharing some similarities at the electrophysiological level, the FRN and RewP 
usually show non-overlapping scalp distributions (i.e. topography), suggesting the existence of partly dissociable neural systems giving rise to them, as we recently confirmed (Gheza et al., 2017).

Evaluative FB processing during PM also influences non-phase locked EEG activities that cannot be captured using a standard ERP analysis (Cohen, 2014). Among them, frontal midline theta (FMT, 4$8 \mathrm{~Hz}$ ) measured at the same recording sites as the FRN and during a similar time window ( $200-400 \mathrm{~ms}$ post-feedback onset) corresponds to a slow oscillation aggregating mostly the phase-locked activity reflected by the FRN (as well as its neighboring positivities, such as P2 and P3) as well as a non-phase locked (induced) component (Cohen and Donner, 2013; Hajihosseini and Holroyd, 2013). Unlike the FRN which has been put forward as a signed RPE signal (Holroyd and Coles, 2002; Ullsperger, 2017), FMT is thought to reflect an unsigned electrophysiological signal that captures dynamic interaction effects between medial frontal cortex (including ACC) and lateral prefrontal areas. Compatible with this view, its power is usually enhanced when cognitive control is needed (Cavanagh et al., 2010; Cavanagh and Shackman, 2015; Cohen et al., 2007; Cohen and Donner, 2013; Hajihosseini and Holroyd, 2013), or higher cognitive effort and task demands are required (Mussel et al., 2016; Wascher et al., 2014). Besides this cognitive control signal represented by FMT, evaluative FB processing usually influences the spectral content of the EEG signal in at least two other non-overlapping bands. The power in the Delta band $(0-4 \mathrm{~Hz})$, measured at central and posterior-parietal sites, usually increases for rewarding compared to non-rewarding conditions (Webb et al., 2017). Last, in the Beta-gamma range (from 20 to $35 \mathrm{~Hz}$ ) at fronto-central sites, (monetary) reward is also associated with increased power (Cohen et al., 2007; Marco-Pallares et al., 2008; Mas-Herrero et al., 2015). The link between power changes in Beta-gamma activity and reward was substantiated by studies showing effects of reward probability (HajiHosseini et al., 2012) and reward magnitude (Marco-Pallares et al., 2008) in this specific frequency band.

Whereas feedback valence and expectation strongly influence the expression of these different feedback-based electrophysiological effects (Ullsperger et al., 2014a), as reviewed here 
above, it is nowadays much less clear to which extent the cost associated with effort anticipation also does, and if so, for which of them and in which direction. Specifically, to which extent the evaluation of a given outcome is shaped by effort anticipation has never been investigated at the electrophysiological level. This paucity is somewhat surprising given that effort is profoundly linked to reward processing. As mentioned above, recent theoretical models advocate their integration in decision making, both in animals (Salamone et al., 2012, 2007, 2003) and in humans (Apps et al., 2015; Kool et al., 2010), corroborating the assumption that PM, and more generally CC, might exploit specific incentive signals or values where both reward and effort/cost have been integrated with one another. In particular anticipated reward and effort rely on a similar cortico-limbic network (Vassena et al., 2014), and are integrated (at the ACC level) during decision making so that the value of an option decreases as a function of associated effort (Croxson et al., 2009; Prévost et al., 2010). These studies suggest that reward processing during PM may be influenced by effort or cost, and more specifically its prospect or anticipation. Moreover, according to some recent models (Pizzagalli, 2014), the most prevalent emotional illness in Western developed countries, namely Major Depressive Disorder (MDD), is thought to be associated with abnormal dopaminergic (DA) signaling in specific corticostriatal networks. Yet, these alterations do not seem to affect hedonic reactions per se (i.e. "liking"; Berridge et al., 2010; Salamone et al., 2007). Instead, they appear to alter incentive salience and reward learning (Admon and Pizzagalli, 2015; Whitton et al., 2016), in interaction with an abnormal stress reactivity (Pizzagalli, 2014). This impairment might also account for the blunted motivation to approach rewarding or pleasurable stimuli (wanting) in these patients, or alternatively engage effort to do so (Salamone and Correa, 2012; Treadway et al., 2012). Further, according to a recent neurocomputational model (Holroyd and McClure, 2015; Holroyd and Umemoto, 2016) the ACC, which provides the main generator of the FRN and FMT oscillations (Smith et al., 2015), is deemed responsible for selecting and motivating extended behavior (see also Holroyd and Yeung, 2012). The ACC would serve as the main node within a hierarchical neural system that translates reward evaluation into CC, implemented in dorsolateral prefrontal areas. Following this model's tenets, 
control signals in the form of FMT oscillations may be generated at the ACC level, as a function of both the learned value and the effort required by the selected, reinforced behavioral response. In this study, we sought to test these predictions, and assess the extent to which the different electrophysiological components described here above could show systematic amplitude variations depending on cost anticipation. More precisely, FMT was expected to increase during the anticipation of effort, due to its putative role in signaling the need for increased control to dorsolateral prefrontal areas, which ultimately coordinate and implement the appropriate behavior. On the other hand, the main ERP components of reward processing (FRN and/or RewP) which are generated in the ACC, might therefore also capture a rapid integration of reward with effort or cost anticipation, given that previous neuroimaging studies pinpointed the ACC as one of the brain regions where this integration took place (Chong et al., 2017; Kurniawan et al., 2013; Vassena et al., 2014).

To this aim, we capitalized on a previously validated gambling task (Hajcak et al., 2005; Paul and Pourtois, 2017) allowing to manipulate on a trial by trial basis FB outcome (either reward or no reward) and reward expectation (being high, intermediate or low) in a factorial design, and eventually measure clear-cut FMT power, FRN, RewP components as well as centroparietal Delta and Betagamma power changes elicited by evaluative FB. Critically, we added a new experimental condition to this paradigm (here below referred to as "special trials") where participants were occasionally invited to redo the gamble in case the outcome turned out to be "no reward" (with the hope for them to transform this no-reward event into a rewarding one). However and noteworthy, in this condition, if they freely decided to do so, they actually had to perform another unrelated task (before being allowed to actually redo the gamble) that clearly included effort expenditure, namely a random dot clicking task (for a similar approach, see Klein et al., 2005; Sherdell et al., 2012; Treadway et al., 2012). Importantly, information about the possibility to redo the gamble (or not) was always provided to participants at the beginning of each trial to activate a specific motivational set throughout the trial (i.e., anticipated cost). After extensive piloting (see Methods and Supplementary Materials - Table 1), we devised specific parameters for this additional dot clicking task to provide an optimal tradeoff 
between effort and reward for our current research goals: preferably, (healthy adult) participants would most of the time choose to redo the gamble (with the goal to win a small monetary reward) despite the need to carry out this orthogonal effort-based clicking task, allowing us to explore reward processing when cost was anticipated or not. Because cost anticipation here refers to the effort required by the additional task, as well the time and mental resources allocated to its execution, it also relates to opportunity cost (Kurzban et al. 2013). This way, we were able to eventually compare evaluative feedback processing between two conditions that were carefully matched along all possible dimensions (e.g., stimulus properties, actual choice and reward probability), but differed regarding cost anticipation (being present or absent). Based on the literature reviewed here above, we formulated different predictions. (i) We surmised a lower FRN for no-reward outcome that could be redone (special trials), compared to the same outcome without this possibility being offered (regular trials), in line with the putative link assumed between this specific ERP component and RPE (Holroyd and Coles, 2002). In other words, the (motivationally relevant) prospect of changing a negative into a positive outcome, albeit requiring extra efforts, would lead to less negative valence, compared to the same condition where no such change could be achieved. (ii) In agreement with the Hierarchical reinforcement learning theory outlined here above (Holroyd and Umemoto, 2016), FMT power, being closely related to CC, should increase for no-reward outcome in the special compared to regular trials, as the latter entailed the prospect of an effortful task. Since the RewP, centroparietal Delta and Betagamma power were primarily related to monetary reward processing per se in previous EEG studies (i.e. in the absence of change in motivation; Bernat et al., 2015; Mas-Herrero et al., 2015; Webb et al., 2017; Weinberg and Shankman, 2017) we performed a systematic exploratory analysis on each of them. If confirmed, these results would therefore lend support to the assumption that these electrophysiological effects during feedback-based PM not only reflect reward processing (in conjunction with expectation), but also cost anticipation. 


\section{Methods}

\section{Participants}

Twenty seven undergraduate students from Ghent University (with normal or corrected-tonormal vision and no reported history of neurological or psychiatric disorders) freely participated in this experiment. Sample size was determined a priori based on an earlier EEG study that used a similar sample size and found clear cut amplitude modulations of FRN and FMT as a function of FB outcome and reward expectation with this same gambling task (Paul and Pourtois, 2017). They all gave written informed consent prior to the start of the experiment and were compensated about $30 €$ for their participation. This amount could be slightly lower (minimum 27.40€) depending on actual task performance (see below). The study was approved by the local ethics committee. One participant was excluded from further analyses due to the unexpected encounter of sickness during EEG recording. Hence, the total sample included 26 participants ( 21 females, age: $M=24.1$ years, $S D=5.4$ ).

\section{Stimuli and task}

We used a gambling task that was previously validated (Hajcak et al., 2007). On each and every trial, participants had to choose one out of four doors by pressing with their right index finger the corresponding key on a response box. After a fixation dot (700 ms) this choice was followed by either positive FB (green "+"), indicating a win of 5 cents, or neutral no-reward FB (red "o") (1000 ms). At the beginning of each trial, participants were informed about reward probability with a visual cue (600 $\mathrm{ms})$, followed by a fixation dot $(1500 \mathrm{~ms})$. The cue was presented in the form of a small disk (pie chart) presented at fixation. Either one, two or three quarters were filled (black/white) corresponding to a reward probability of respectively 25,50 or $75 \%$. Because four doors were presented for the choice, a $25 \%$ reward probability therefore indicated that only one door actually contained the reward, two doors in the case of $50 \%$ reward probability and three doors for $75 \%$ reward probability. Unbeknown to participants, the outcome was actually only related to these probabilities (and not their actual choices), ending up with a preset winning of $€ 12.40$. Inter trial interval was fixed and set to $1000 \mathrm{~ms}$. 
Hence, by crossing the three possible reward probabilities with the two opposite outcomes, six trial types were included in a factorial design. These six trial types were deemed "regular" and did not involve any specific effort or motivational component. Anticipated cost was low for them. To ensure participants paid attention to the cue and outcome, we also used catch trials randomly interspersed in the trial series (for a similar procedure, see Paul and Pourtois, 2017). Catch trials were identical to regular trials, except that a specific probe appeared either after the cue or FB. More specifically, in 24 trials at the cue offset this probe asked participants to report the winning chance ("How many doors contain a prize?", allowing responses from 1 to 3 ). In 24 different trials, they were asked about the expectedness of the outcome at FB offset, and answers were collected by means of a visual analog scale (VAS).

Besides regular trials, we also introduced "special" trials (i.e. they included a motivational component), where anticipated cost was transiently induced, selectively. Special trials only included $50 \%$ reward probability (maximum uncertainty) and were rewarded with 5 cents as well. Regular and special trials were shown in random order (for any reward probability and outcome condition). Special trials differed from regular trials by means of a specific visual cue lasting $1000 \mathrm{~ms}$ and informing participants about the start of this "special" case. After this cue, trial structure of special trials was identical to regular trials, with the exception that the four doors were displayed in green color (as opposed to white color for regular trials) to remind participants of this special case throughout. After the choice, if the FB turned out to be a reward (50\%), then the trial terminated. However, if the FB turned out to be no-reward $(50 \%)$, a second choice was submitted to the participants. They were invited to choose between two options: either to re-do this specific gamble or to carry on with a new gamble. If they decided to redo it, reward magnitude was increased to 10 cents. Hence, if they decided to redo it, they knew they might transform the no-reward just received - last gamble - to a possible rewarding outcome - new gamble within the same trial. We chose this specific reward magnitude to balance the maximum payoff between regular ( 5 cents/one gamble) and special trials (10 cents/two gambles). Nevertheless, because this gamble also had a $50 \%$ reward probability, reward uncertainty 
was still high. No time limit was imposed for this second choice. If they decided not to redo the gamble, then the trial terminated and they moved on to the next one. However and crucially, if they opted to redo the gamble, they were asked to complete another unrelated task first, namely a random dot clicking task (Klein et al., 2005; Sherdell et al., 2012; Treadway et al., 2009). This extra task therefore served as "stake" and involved an effort component. We devised this task to provide an ecological effort requirement that had both a physical and attentional part, as opposed to physical only (e.g. effortful gripping; Kurniawan et al., 2010; Pessiglione et al., 2007) or mental only (e.g. arithmetic calculation; Vassena et al., 2014). This random dot clicking task resembled the common bothering activity of closing pop-up windows while internet-browsing; it required a sustained activity (around 8 seconds) that participants deemed bearable most of the time, being driven by the prospect of an extra reward. Specific parameters defining the duration of the dot clicking task, as well as the probabilistic reward in redo-trials (10 cents), were selected after extensive pilot testing. These parameters allowed to set an optimal cost/benefit tradeoff (i.e. positive re-do choices > $50 \%$; see Results; pilot data are reported in Supplementary materials - Table 1). For special trials, we explicitly chose a $50 \%$ reward probability condition to provide a balanced amount of reward and no-reward trials, which eased considerably data analyses and enabled us to avoid asymmetries in the signal-to-noise ratio between conditions.

This random dot clicking task was designed as follows: a small cross ("+", 1x1 mm) appeared at a random position on the screen until the participant clicked on it using the mouse, with 8 iterations of this task at 8 different locations (randomly selected at each iteration within a randomly generated list of 100 coordinates). Upon completion of the 8 successive clicks, a 500 ms screen announced the start of the redo-gamble, with the trial structure being identical to what is described here above (see regular trials). Redoing the gamble resulted in either no reward (50\%) or (10 cents) reward (50\%). An additional amount varying between 0 and $2.60 €$ could be won with these special trials. 
All stimuli were shown against a grey homogenous background on a 21-in CRT screen and controlled using E-Prime (V 2.0, Psychology Software Tools Inc., Sharpsburg, PA). At the end of the experiment participants were asked to rate the pleasantness and difficulty of the random dot clicking task using a continuous VAS, as well as their actual motivation to carry out it in order to redo the gamble. Additionally, they were asked to rate the pleasantness of the rewarding and no-rewarding FB, separately for regular (either 5 or 0 cent) and redo trials (following the clicking task - either 10 or 0 cent).

[Figure 1 about here]

\section{Procedure}

After reading the instructions, participants were first familiarized with fourteen practice trials of the gambling task, including three special trials (two of them providing no-reward, thus allowing familiarization with the occurrence of a second choice now and then, and the random dot clicking task). In total, 392 trials were regular. One hundred and four trials were special. Given that special trials always involved a $50 \%$ reward probability, the choice to perform the random dot clicking task (and to redo the gamble) was eventually submitted 52 times to them in total. For FRN analyses, common practice suggests using at least 20 trials per condition (Marco-Pallares et al., 2011). With these considerations in mind, for the $25 \%$ reward probability condition, 144 trials were used, of which 108 with no-reward FB and 36 with reward FB. The reverse was obtained for the $75 \%$ reward probability condition. Last, for the $50 \%$ reward probability condition, 104 trials were used, half being rewarding $(n=52)$ and the other half non-rewarding $(n=52)$. The same trial type could be presented consecutively. The experiment consisted of four blocks comprising a random combination of 124 trials each. After each block, a short break was included and participants were informed about their current (cumulative) payoff. 


\section{Recording and Preprocessing of Electrophysiological Data}

EEG was recorded using a 64-channel Biosemi Active Two system (http://www.biosemi.com) with four additional electrodes measuring horizontal and vertical eye movements. EEG was sampled at $512 \mathrm{~Hz}$ and referenced to the Common Mode Sense (CMS) active electrode and Driven Right Leg (DRL) passive electrode. The EEG was preprocessed offline with EEGLAB 13.5.4b (Delorme and Makeig, 2004), implemented in Matlab R2012b. A $0.05 / 35 \mathrm{~Hz}$ high/low pass filter was applied after rereferencing the EEG signal to the averaged mastoids. An independent component analysis was run on the continuous data. Individual epochs were extracted from -2000 to 2000 ms around FB onset and a pre-feedback baseline was subtracted (-250 to 0). Artefactual ICA components were manually selected focusing on eye artifacts and spatial or temporal discontinuities. A semi-automatic artefact correction procedure was applied to eliminate trials with voltage values exceeding $\pm 90 \mu \mathrm{V}$ or slow voltage drifts with a stronger slope than $\pm 90 \mu \mathrm{V}$, as well as based on visual inspection. For each subject separately, artefact-free epochs were grouped according to the six regular and two special conditions. Regular trials included expected, no-expectations and unexpected ${ }^{1} \mathrm{FB}$ associated with reward (deriving from $75 \%, 50 \%, 25 \%$ reward probability trials respectively), and expected, no expectations and unexpected FB associated with no-reward (deriving from $25 \%, 50 \%, 75 \%$ reward probability trials respectively). Special trials involved only no-expectations FB, providing either reward or no-reward (from 50\% reward probability special trials). To overcome getting different signal to noise ratios between conditions (Keil et al., 2014), the same number of trials (randomly sampled) was used for all of them, being defined subject-wise based on the condition with the lowest trial count.

The FRN was quantified at FCz as the difference between the most negative peak (N200: within $200-350 \mathrm{~ms}$ ) and the average voltage of the preceding and following positive peaks (P170: within 150 - $250 \mathrm{~ms}, \mathrm{P} 300$ : within 250 - $600 \mathrm{~ms}$ ), to control for possible confounding effects of the positive components surrounding the N200, as often performed in ERP studies (Chase et al., 2011; Oliveira et

\footnotetext{
${ }^{1}$ These labels refer here to the objective reward probability and not the subjective expectation.
} 
al., 2007; Sallet et al., 2013; Yeung and Sanfey, 2004). The RewP was defined as the average amplitude at $\mathrm{Cz}$ and $\mathrm{FCz}$ within the $235-285 \mathrm{~ms}$ interval post-feedback onset, corresponding to the $50 \mathrm{~ms}$ window surrounding the peak of the difference reward - no-reward (Novak and Foti, 2015; see Figure 3).

Time frequency analysis was done using EEGLAB built-in std_ersps() function, based on complex Morlet wavelet convolution (2 to 8.75 cycles, 0.8 to $35 \mathrm{~Hz}, 75$ log spaced frequencies, 200 time points per epoch). The time interval -500 to -200 ms before FB onset was used for baseline normalization. FMT band power change $(4-8 \mathrm{~Hz})$ were defined as the mean within $200-400 \mathrm{~ms}$, decibel $(\mathrm{dB})$ converted $(10 * \log 10[$ power/baseline]) at FCz. The same approach was adopted for the estimation of Delta $(0.8-3.9 \mathrm{~Hz})$ and Beta-gamma $(20-35 \mathrm{~Hz})$ band power changes, defined respectively as the mean amplitude within $200-400 \mathrm{~ms}$ at a set of parietal sites (CPz, CP1, CP2, CP3, CP4) for Delta, and within 250 - 350 ms at a set of frontocentral sites (FCz, Fz, FC1, FC2) for Betagamma. Time windows ${ }^{2}$ and channel locations were based on the band-specific maximal power from the grand average of all conditions in regular trials (Luck and Gaspelin, 2017; see Figures 4-6).

\section{Data Analysis}

For all analyses, significance alpha cutoff was 0.05. At the behavioral level, our main dependent variable was the number of redo (expressed in percentage) associated with special trials. For the catch trials at the cue level, the amount of correct responses was converted to percentage. At the FB level, the subjective ratings were first transformed to percentages, arbitrarily setting one anchor ('very unexpected') to 0 and the other one ('very expected') to 100 . These ratings were considered to be correct if the given value fell within a $\pm 20 \%$ range around the correct response $(25,50$ or $75 \%$. See

\footnotetext{
${ }^{2}$ As visible from Figures 4 and 5 (panel B), the estimated power in the Theta and Delta bands peaked around $300 \mathrm{~ms}$ following FB onset, demarcated by the 0 time point in these plots. Because the time/frequency convolution used assesses non-phase-locked event-related changes in the ongoing oscillatory activity, they can develop and peak rapidly after the time-locking event, even before a full period in a given frequency is actually completed (see also Cohen, 2014).
} 
Paul and Pourtois, 2017). Similarly, post-experiment VAS ratings of pleasantness of the FB (for regular and redo trials), as well VAS ratings of the random dot clicking task, were transformed to percentages setting anchors to the boundaries of the scales.

At the electrophysiological level, two sets of statistical analyses were performed. First, using the regular trials only, we assessed amplitude changes (FRN, RewP, FMT, Delta and Beta-gamma power) depending on reward probability and outcome. To this aim, repeated measures ANOVAs with FB expectation (expected, no expectations, unexpected) and outcome (reward vs. no-reward) as within-subject factors were performed. The trial count was equal across the six main conditions (regular trials): $\mathrm{M}_{\text {subject }}=27.0, \mathrm{SD}_{\text {subject }}=4.0$. Next, we compared FRN, RewP, FMT, Delta and Betagamma power for the no expectations condition only (i.e. 50\% reward probability) between regular trials (no effort anticipation) and special trials (effort anticipation). To this aim, special trials that led to a no-reward and were eventually not redone by the participants (corresponding to a low number, see results below for actual proportion) were discarded from this analysis. Hence, with this second analysis, we could compare at the electrophysiological level the exact same outcome (either reward or no reward, with the same probability) when anticipated effort was absent vs. present (and eventually exerted). In this analysis, for each ERP component separately, a repeated measure ANOVA was carried out with trial type (special vs. regular) and outcome (reward vs. no-reward) as withinsubject factors. The trial count was equal across the four main conditions (special vs. regular trials, with two outcome levels each time): $\mathrm{M}_{\text {subject }}=36.3, \mathrm{SD}_{\text {subject }}=7.3$.

Further exploratory Bayes Factor analyses (Rouder et al., 2017) were carried out with the JASP software package (JASP Team, 2017) with the default prior settings. 


\section{Results}

\section{Behavioral Results}

The accuracy for the cue ( $\mathrm{M}$ correct= $84.5, \mathrm{SD}=11.5$ ) and for the outcome (FB expectation: $\mathrm{M}$ correct $=69.5, S D=18.4)$, as inferred from the catch trials, were well above chance level and compatible with a previous study where the same gambling task was used (Paul and Pourtois, 2017). Overall, for special trials, participants chose to redo the gamble most of the time (\% yes: $M=79.8, S D$ $=30.97$ ). However, five participants chose to re-do the gamble seldom ( $\%$ yes: $M=21.9, S D=11.7$ ) and were deemed outliers (based on mean \pm 1.5 standard deviations criterion). Accordingly, we excluded them from the subsequent analyses. On average, $1036 \mathrm{~ms}$ (SD = $853 \mathrm{~ms}$ ) elapsed after no-reward FB before participants chose to redo the gamble (special trials). Post-experiment ratings confirmed that participants ( $n=21$ after removing the five outliers) reported to be motivated to carry out the random dot clicking task $(\mathrm{M}=80.2 \%, \mathrm{SD}=15.5)^{3}$ in order to re-do the gamble. By comparison, a much lower motivation was observed for the five participants excluded $(M=42.3 \%, S D=35.2)$. Hence, there appeared to be a clear association between choosing to redo the gamble and be exposed to effort expenditure during the random dot clicking task, and its subjective evaluation in terms of motivation. Further, the ratings for the random dot clicking task showed that it was evaluated as being neutral and relatively easy (pleasantness: $M=48.5 \%, S D=29.7$; difficulty: $M=20.0 \%$, SD $=20.1$ ). Last, no significant differences were found between the evaluation of the reward FB after the regular (5 cent) and the redo trials (10 cent. $t(20)=-1.139, p=.268$, Cohen's $\left.d_{z}=-0.25\right)$, nor between no-reward after the regular and the redo trials $\left(t(20)=1.254, p=.224\right.$, Cohen's $\left.d_{z}=0.27\right)$.

\section{Electrophysiological Results}

In the first set of analyses, we assessed the effects of FB expectancy and FB outcome on each electrophysiological marker separately, using regular trials only. The analysis performed on the FRN

\footnotetext{
${ }^{3}$ Ratings of motivation and difficulty about the random dot clicking task were not reported by 3 participants. Related data refer to 18 out of 21 participants complying with exclusion criteria.
} 
amplitudes showed a significant main effect of expectation $\left(F(2,50)=8.55, p=.001, \eta^{2} p=.255\right)$, outcome $\left(F(1,25)=16.38, p<.001, \eta^{2} p=.396\right)$ and an interaction between these two factors $(F(2,50)$ $\left.=5.62, p=.006, \eta^{2} p=.184\right)$, confirming its compatibility with the RPE framework. The FRN component was larger for no-reward compared to reward FB ( $\mathrm{M}$ reward $=-9.42, \mathrm{SE}=0.72, \mathrm{M}$ no-reward $=-12.38$, $\mathrm{SE}=1.00)$ and unexpected compared to expected. Simple main effects of expectation were significant for no-reward $\left(F(2,24)=9.98, p=.001, \eta^{2} p=.454\right)$, but not for reward $F B(F(2,24)=0.58, p=.569)$. The analysis performed on FMT power showed a significant main effect of expectation $(F(2,50)=$ 16.06, $\left.p<.001, \eta^{2} p=.391\right)$, and outcome $\left(F(1,25)=8.64, p=.007, \eta^{2} p=.257\right)$, without a significant interaction between these two factors $(F(2,50)=0.31, p=.732)$. The analysis performed on the RewP amplitudes showed a significant main effect of FB expectation $\left(F(2,50)=10.12, p<.001, \eta^{2} p=.288\right)$ and outcome $\left(F(1,25)=57.22, p<.001, \eta^{2} p=.696\right)$, without significant interaction between these two factors $(F(2,50)=1.04, p=.360)$. The RewP was larger for reward compared to no-reward FB (M reward $=11.27, \mathrm{SE}=1.43, \mathrm{M}$ no-reward $=5.06, \mathrm{SE}=1.35)$ and for unexpected compared to no-expectation and expected FB, with a significant decrease in amplitude with increasing expectation (linear contrast, $\left.F(1,25)=10.90, p=.003, \eta^{2} p=.304\right)$. Similarly to the RewP, the analysis performed on centroparietal Delta power showed a significant main effect of FB expectation $\left(F(2,50)=12.17, p<.001, \eta^{2} p=.327\right)$, and outcome $\left(F(1,25)=20.75, p<.001, \eta^{2} p=.454\right)$, without significant interaction between these two factors $(F(2,50)=3.09, p=.054)$. Finally, the analysis performed on frontal Beta-gamma showed a trend for the main effect of $\mathrm{FB}$ outcome $\left(F(1,25)=3.57, p=.070, \eta^{2} p=.125\right)$, with higher power values for reward $(M=0.77, S E=0.23)$ compared to no-reward $F B(M=0.42, S E=0.14)$. Notably, FB outcome had therefore opposite effects on these non-overlapping frequency bands, with higher FMT power for no-reward vs reward FB, but conversely higher Delta and Beta-gamma power for reward vs no-reward FB (see Figures 4 and 5).

In the second set of analyses, we assessed the effects of trial type and FB outcome, using special trials followed by a positive redo-choice and regular trials with the same reward probability - $50 \%$. We first tested the hypothesis that the FRN amplitude might be lower for special no-reward (when the 
choice to redo was actually made, see methods and results above) compared to regular no-reward FB. This analysis showed a significant main effect of trial type $\left(F(1,20)=7.34, p=.013, \eta^{2} p=.269\right)$ and $\mathrm{FB}$ outcome $\left(F(1,20)=8.76, p=.008, \eta^{2} p=.305\right)$. The interaction between these two factors was not significant $(F(1,20)=1.50, p=.236)$. The FRN was larger for no-reward compared to reward $\mathrm{FB}$, and for special trials compared to regular trials. This latter difference was significant for reward $(F(1,20)=$ 9.21, $p=.007, \eta^{2} p=.315 . \mathrm{M}$ regular $=-8.88, \mathrm{SE}=0.94, \mathrm{M}$ special $\left.=-10,54, \mathrm{SE}=0.99\right)$ but not for noreward FB $(F(1,20)=0.55, p=.465)$ (Figure 2). Next, we tested the hypothesis that FMT power was larger for special no-reward (when effort was anticipated) compared to regular no-reward. This analysis showed a significant main effect of FB outcome only $\left(F(1,20)=16.15, p=.001, \eta^{2} p=.447\right)$, with higher values for no-reward compared to reward FB. The main effect of trial type was not significant $(F(1,20)=1.69, p=.208)$. No significant interaction between trial type and $F B$ outcome was found $(F(1,20)=0.07, p=.795)$ (Figure 4). Hence, these two analyses failed to confirm the prediction that a second choice might influence (i.e. decrease) the processing of the negative valence of the FB (FRN and RPE), or that effort anticipation might increase cognitive control ${ }^{4}$ (FMT power).

However, and interestingly, reward processing per se seemed to be influenced by the manipulation of effort anticipation, as suggested indirectly by the FRN data analysis reported here above. To test this possibility, we analyzed the RewP ERP component using the same statistical model. This analysis showed a significant main effect of trial type $\left(F(1,20)=8.54, p=.008, \eta^{2} p=.299\right)$ and $\mathrm{FB}$ outcome $\left(F(1,20)=55.89, p<.001, \eta^{2} p=.736\right)$. The RewP was larger for reward compared to noreward FB, and for special compared to regular trials. Importantly, this analysis also showed a significant interaction between trial type and FB outcome $\left(F(1,20)=6.82, p=.017, \eta^{2} p=.254\right)$. As suggested indirectly by the FRN data analysis reported here above, the RewP was significantly larger for special compared to regular trials when the FB was rewarding $\left(F(1,20)=9.54, p=.006, \eta^{2} p=.323\right.$.

\footnotetext{
${ }^{4}$ We also explored possible changes occurring at the cue level between the two main conditions (see Supplementary Materials for numerical values and statistical results). Interestingly, FMT was larger for special than regular trials, likely suggesting some enhanced CC cue-based for the former compared to the latter ones. By comparison, the CNV component (ERP) was similar between these two conditions, suggesting that anticipation of the upcoming door selection task was balanced between them.
} 
$\mathrm{M}$ regular $=10.54, \mathrm{SE}=2.00, \mathrm{M}$ special $=13.57, \mathrm{SE}=1.97)$ but not when it was not $(F(1,20)=1.92, p=$ .182) (Figure 3). Noteworthy, the analysis performed on centroparietal Delta power showed a similar effect, with a significant main effect of trial type $\left(F(1,20)=8.56, p=.008, \eta^{2} p=.300\right)$, a significant main effect of FB outcome $\left(F(1,20)=12.43, p=.002, \eta^{2} p=.383\right)$, and the interaction between FB outcome and trial type approaching significance $\left(F(1,20)=4.14, p=.055, \eta^{2} p=.171\right)$. Delta power values were larger for reward compared to no-reward FB, and for special compared to regular trials, with this latter difference being significant for reward $\left(F(1,20)=13.10, p=.002, \eta^{2} p=.396\right)$, but not for no-reward FB $(F(1,20)=0.89, p=.358)$ (Figure 5). Finally, the analysis performed on frontal Beta-gamma power showed a significant main effect of FB outcome $\left(F(1,20)=6.57, p=.019, \eta^{2} p=.247\right)$ and importantly, a significant interaction between trial type and FB outcome $\left(F(1,20)=4.80, p=.040, \eta^{2} p=.194\right)$. Betagamma oscillations increased for reward compared to no-reward FB. Interestingly, this latter difference was significant for special trials $\left(F(1,20)=12.22, p=.002, \eta^{2} p=.379\right.$. $\mathrm{M}$ reward $=1.02, \mathrm{SE}=$ $0.26, \mathrm{M}$ no-reward $=0.10, \mathrm{SE}=0.18)$ but not for regular ones $(F(1,20)=1.67, p=.212 . \mathrm{M}$ reward $=$ $0.63, \mathrm{SE}=0.29, \mathrm{M}$ no-reward $=0.24, \mathrm{SE}=0.19)($ Figure 6$)$.

As suggested by the RewP and beta-gamma results reported here above (as well as Delta to a lesser degree), reward processing was increased when effort expenditure could be avoided. Accordingly, it is conceivable that the five subjects who chose the re-do the gamble seldom (and were excluded from the analyses) might show an equal or even stronger gain in reward processing when effort could be avoided since their behavior translated effort avoidance. To test this assumption indirectly, we performed auxiliary Bayesian factor analyses using the full sample ( $n=26$ participants). For each electrophysiological marker of reward processing separately (RewP ERP component, Delta and Beta-gamma power), we tested by means of a Bayesian paired samples t-test the strength of the evidence in favor of the alternative hypothesis that posited a larger amplitude value for reward processing in special (where effort anticipation was induced) compared to regular trials (where it was absent). Results showed that the alternative hypothesis was $39.5,41.8$, and 1.4 times more likely than the null for RewP, Delta and Beta-gamma power, respectively. These results provide thus very strong 
evidence in favor of an increase in reward processing at the RewP and Delta power levels when effort could be avoided.

Finally we explored, across the whole sample $(n=26)$, if the percentage of redo correlated with these electrophysiological effects. For each electrophysiological marker of reward processing separately, we first computed a difference-score by subtracting the mean activity for the regular from the special trials. Non-parametric correlations by means of Spearman's Rho were used. However, these correlations failed to reveal significant effects (RewP: $r_{s}=-0.105, p=0.611$; Delta: $r_{s}=0.247, p=0.225$; Beta-gamma: $\left.r_{s}=0.319, p=0.112\right)$. To note, the percentage of redo was high for the whole sample, with 11 participants that redid the gamble $100 \%$ of the time, hence the inter-individual variability was low for this metric.

\section{Discussion}

To explore modulatory effects of cost anticipation on PM and reward processing, we used a previously validated gambling task in combination with a random dot clicking task (deriving from the work of Sherdell et al., 2012; and Treadway et al., 2009). To this end, two trial types, shown in random order, were used and compared: regular trials that did not involve cost anticipation, and special ones where a random dot clicking task could be carried out in case of no-reward as outcome, with the hope for the participants to transform this worse than expected event into a rewarding one. As a result, cost anticipation was increased in the latter compared to former trials, while all other dimensions were kept similar. A number of important new results emerge from this study. Cost anticipation reliably influenced the RewP and Delta (as well as Beta-gamma) power, but not the FRN and FMT, suggesting a direct influence on reward processing (as opposed to RPE signals captured by the FRN, or the need for $\mathrm{CC}$, as reflected by FMT). More specifically, using these well-established neurophysiological markers of reward processing (RewP, Delta and Beta-gamma power), we found evidence for each of them for a systematic enhancement of this reward-based processing at the FB level when cost 
anticipation was activated, but the choice and ensuing dot clicking task could eventually be avoided. The uncertain outcome of the first gamble turned out to be rewarding, precluding in turn to perform a second choice and importantly, cancelling the second choice and random dot clicking task.

Our first set of analyses (regular trials) confirmed that FRN was sensitive to RPE (Holroyd and Coles, 2002; Walsh and Anderson, 2012), which was a pre-requisite to assess subsequently effects of cost anticipation on it, in special trials. Unexpected no-reward FB yielded the largest FRN, as dominant models of PM would predict (Ullsperger et al., 2014a, 2014b). Further, no-reward and unexpected FB led to substantial FMT power increases compared to rewarding and expected FB, replicating previous EEG findings obtained with the same gambling task (Hajcak et al., 2007; Paul and Pourtois, 2017; see also Sallet et al., 2013). Although FRN and FMT power showed similar effects during reward processing at the FB level, yet these two markers did not fully overlap, and hence they might reflect different processes. Unlike the FRN, FMT power did not show a significant interaction between outcome and reward expectation, which is consistent with the assumption that spectral perturbations in this specific frequency band over medial frontal areas reflect signed RPEs only indirectly, especially when the evoked and induced activities are not disentangled from one another (Hajihosseini and Holroyd, 2013). Presumably, the total FMT power captures expectation's violation, conflict detection and/or the need for CC, rather than RPE per se or exclusively (Cavanagh et al., 2012; Cavanagh and Frank, 2014; Cohen and Donner, 2013). Interestingly, RewP, Delta and Beta-gamma power had opposing valence effects and non-overlapping scalp distributions compared to the FRN and FMT, showing larger amplitudes at central, centro-parietal and fronto-central electrodes for reward than no reward FB. RewP and Delta also showed increases in signal strength with increasing (reward) uncertainty, while Beta-gamma did not.

At the behavioral level, we found that the parameters chosen (see supplementary materials section for details) eventually created an optimal tradeoff between effort exertion (i.e. random dot clicking task) and the extra reward prospect to allow us to explore reward processing at the EEG level 
when cost anticipation was elicited: for special trials, the majority of participants (i.e., 21 out of 26 included in the sample) eventually chose to redo the gamble most of the time upon the experience of an unexpected no-reward outcome in these special trials, hence they decided to perform the auxiliary random dot clicking task and thereby exerted efforts, translating (enhanced) incentive motivation (Berridge and Robinson, 2003, 1998). Notwithstanding the presence of cost and effort anticipation in these trials, at the EEG level, we failed to observe an increase of FMT power, however. Similarly, despite the overall preference toward the opportunity to undo the loss, we failed to observe a decrease of FRN amplitude in the same condition. Nonetheless, when considering direct electrophysiological markers of reward processing (as opposed to RPE for FRN, or the need for CC in the case of FMT power), namely RewP, centroparietal Delta and frontocentral Beta-gamma power, we found that cost anticipation did reliably modulate their amplitudes, suggesting the timely integration of reward and effort/cost anticipation during evaluative FB processing. While this interaction effect was at trend level only for the Delta power, it was clearly found when considering the RewP ERP component and Beta-gamma power changes: these markers were substantially increased for rewarding FB when comparing special to regular trials, hence when comparing trials with vs. without cost anticipation. In other words, participants appeared to assign more hedonic or positive value to the rewarding FB when it signaled that extra effort expenditure was precluded (special trials), compared to a control condition where anticipated effort was always absent/omitted (regular trials), corroborating the assumption that cost anticipation and reward signals were timely integrated at this level. Because reward expectation was intermediate (50\%) and balanced between these two conditions, this variable cannot account for this effect. Likewise, reward magnitude was matched between them, ruling out the possibility that this factor could explain this modulatory effect. Moreover, an auxiliary data analysis based on Bayes factors and run on the full sample $(n=26)$ confirmed strong evidence in favor of a significant increase in the amplitude of the RewP ERP component as well as Delta power when reward processing was accompanied by the avoidance of this anticipated cost. 
Although the modulation of reward-related effects on FB processing was mainly related to an anticipatory component during special trials, the trial structure did not allow us to parse effort anticipation per se, from a more general cost associated with the consequences of the subsequent choice (i.e. to redo the gamble most of the time). At the time of FB delivery after the first gamble (special trials), participants likely anticipated and integrated not only the need to execute an effortful task, but also the time they would therefore need to spend on it and the second gamble ensuing. Accordingly, the enhanced reward-related activity seen for the first gamble during special trials in case of reward outcome might result from the blend of multiple processes and components. As surmised here above, opportunity cost (Kurzban et al., 2013) is thought to play an important role in this modulation. In the present case (cf. special trials), a cost arose and was likely computed and rapidly integrated with reward because participants anticipated they had to spend some time doing another task (i.e. the dot clicking task) and thus consume mental resources for it, something that precluded their allocation to another valuable task or activity (the gamble). Further, not only did the effortful dot clicking task impeded or interfered now and then with the main task (gambling), it probably also artificially prolonged the total time they had to spend before they could eventually complete the experiment and leave the laboratory. Hence, it appears parsimonious to assume an interaction effect between reward and a more general cost anticipation to explain these results. This general cost likely corresponded to the effort itself as well as the associated opportunity cost. Moreover, given that the analyses performed on reward-related EEG markers were exploratory in nature, we sought to corroborate this conclusion more directly and firmly at the empirical level. To this aim, we ran a control behavioral experiment (see supplementary materials for details) where we used a similar experimental procedure and task (without EEG and including less conditions), but critically, we added now and then subjective ratings of the $\mathrm{FB}$ along specific affective dimensions during task execution. Confirming our interpretation, results of this control behavioral experiment showed that reward FB encountered during special trials was associated with higher levels pleasantness and relief (without changes in frustration however) compared to the same reward FB encountered during regular trials (see Figure 1 
- Supplementary Materials). Combined together, these new results therefore lend support to the assumption that reward and cost anticipation are timely integrated during FB processing. Moreover, they suggest that standard electrophysiological markers of reward processing in humans (namely the RewP ERP component and frontocentral Beta-gamma power changes) track changes in motivation to some extent, and more specifically incentive motivation (assuming that motivation to engage in rewarding tasks or activities critically depends on perceived effort). As such, they are broadly consistent with the ubiquitous principle of economy that rules many facets of human decision making (Botvinick and Braver, 2015; Hull, 1943; Westbrook and Braver, 2016), whereby when effort to gain rewards can be avoided (because of a specific choice made or task configuration/set), reward processing is in turn transiently enhanced.

Our new findings also have important methodological and clinical implications. For example, Anhedonia is usually viewed as a cardinal diagnostic and endophenotypic feature of several emotional disorders, including MDD (Pizzagalli, 2014). Moreover, recently MDD has been associated with blunted RewP and/or posterior parietal Delta power in different EEG studies, suggesting decrease reward processing during gambling in these patients relative to healthy controls (Olbrich and Arns, 2013; Proudfit et al., 2015; Webb et al., 2017; Weinberg and Shankman, 2017; Whitton et al., 2016). As our new EEG results indirectly suggest, amplitude variations of the RewP and posterior parietal Delta can be observed (in healthy controls) when reward expectation is kept constant, but cost anticipation varies systematically across conditions, with effort avoidance clearly increasing reward processing. Intriguingly, a decreased RewP and/or posterior parietal Delta power in MDD might in principle reflect an abnormal integration of reward with cost anticipation, as opposed to decreased reward processing per se. Additional EEG studies are therefore needed to assess the actual contribution of cost anticipation vs. reward (and their joint effect) to amplitude modulations of these electrophysiological markers of reward processing in MDD during gambling and PM. 
Some limitations warrant comments. Presumably, the lack of systematic amplitude modulation of the FRN as a function of the opportunity to undo a loss, as was initially hypothesized, might be partially related to the specifics of our experimental procedure. Because we used monetary reward as main incentive, defensive motivation or negative affect was probably not elicited in case of no-reward outcome. This might account for the lack of systematic amplitude modulation of the FRN or FMT as a function of cost or effort anticipation in the present case. Moreover, although our new results are compatible with the HRL-ACC theory (Holroyd and Umemoto, 2016), here we used a gambling task devoid of learning, a factor which may have reduced artificially the need for CC in case worse than expected outcome was experienced, accounting in turn for the lack of systematic amplitude variations of the FRN and FMT with the elected cost anticipation manipulation. However, the lack of learning was an advantage because we could easily compare at the EEG level special to regular trials without considering specific bins or time intervals. Last, we acknowledge that it would probably have been better to compare two conditions that only differed in terms of effort, being either low or high for example (Vassena et al., 2014). However, we had to include additional conditions in the design in the present case (see regular trials) to ascertain first that the electrophysiological markers under scrutiny were sensitive to feedback expectancy and valence, as previously found. Adding them eventually made the design more complex. However, it was an important pre-requisite at the methodological level. Accordingly, future studies where a more controlled parametrization of cost is achieved are needed to better disentangle which underlying component of cost is causally related to the changes observed in reward processing at the FB level. In this context, the results reported in this study ought to be seen as a first attempt to explore systematically the malleability of standard electrophysiological markers of PM to changes in reward and cost anticipation concurrently.

To conclude, the results of this study show that reward and cost anticipation integrate with one another during FB processing after gambling. Reward processing, as measured using RewP amplitude and frontocentral Beta-gamma power (as well as posterior parietal Delta power), was increased when extra effort could occasionally be avoided, in line with the broad principle of economy 
and the aversiveness of effort anticipation. Moreover, at the subjective level, participants evaluated this FB as more pleasant (and relieving) compared to the same FB provided without any cost component. All in all, these results dovetail with the assumption that incentive motivation (where effort and reward are considered concurrently) dynamically shapes FB processing during gambling. As such, these new results might have implications for identifying stable or reliable electrophysiological markers of Anhedonia, which is usually characterized by both reward-related and motivational impairments (Sherdell et al., 2012; Treadway et al., 2012). Ultimately, systematically exploring changes in reward processing depending on cost anticipation at the ERP and time-frequency levels in MDD patients, as done here in healthy adult participants, could help better disentangle if the observed impairments during FB based reward processing actually stems from abnormal reward processing per se, or instead a complex interaction effect between reward and cost anticipation. 


\section{Acknowledgements}

RDR, CB and GP are funded by a Concerted Research Action Grant from Ghent University. GP is supported in part by a 2015 NARSAD Independent Investigator Grant from the Brain \& Behavior Research Foundation. This work was also supported by the Belgian Science Policy, Interuniversity Attraction Poles program (P7/11). We thank Beatrice Del Frate for her help with the data collection. 


\section{Figures legends}

Figure 1. Overview of the task and trial structure. (A) In regular trials, participants were first informed about reward probability (by means of a black and white pie chart indicating 25,50 or $75 \%$ winning probability, shown in random order). After they picked one door, they received either a reward (5 Euro cent) or no-reward FB, depicted by a green cross or red circle, respectively. (B) In special trials, at the beginning of the trial, a specific cue (i.e. the words "Special situation" written in Dutch) informed participants about the fact that these trials were special compared to the regular ones because reward probability was $50 \%$ only and, more importantly, in case of no-reward FB outcome they could choose to redo the gamble. During the door selection, the color of the doors was marked in green in order to remind them of this special case. If participants chose to redo the gamble after no-reward (which they did on a majority of trials; see results), an orthogonal dot clicking task (including effort expenditure) had first to be carried out before starting the gamble again. Reward magnitude was doubled for the second gamble in case of reward (i.e. 10 Euro cent) to maximize the probability of redoing the gamble after no-reward, as established based on pilot testing (see Supplementary materials).

Figure 2. FRN results. (A) Grand average ERP waveforms computed at FCz for all six main conditions (regular trials). The FRN was computed as the difference between the most negative peak (N200: within $200-350 \mathrm{~ms}$ ) and the average voltage of the preceding and following positive peaks (P170: within 150 - $250 \mathrm{~ms}$, P300: within $250-600 \mathrm{~ms}$ ). (B) A significant interaction between FB outcome and FB expectancy was evidenced for the FRN, whereby it was the largest for unexpected noreward FB. (C) Mean amplitude of the FRN in the $50 \%$ reward probability condition as a function of effort anticipation (absent/regular trials vs. present/special trials) and FB outcome (reward vs. noreward FB). The FRN was significantly larger for no-reward compared to reward FB, and for special compared to regular trials, but without interaction between these two factors. The error bar corresponds to 1 standard error of the mean. 
Figure 3. RewP results. (A) Grand average ERP waveforms from $\mathrm{Cz}$ and $\mathrm{FCz}$ pooled together for the $50 \%$ reward probability condition. The left inset shows the RewP for regular (solid lines) and special (dashed lines) trials, separately for reward (dark blue) and no-reward (light blue) outcome. The right inset shows the corresponding difference waves for the two main conditions (regular trials-black line and special trials-red line) obtained after the ERP activity for no-reward was subtracted from the one corresponding to reward. The RewP was computed as the average amplitude at $\mathrm{Cz}$ and FCz within the 235-285 ms interval post-feedback onset (the corresponding time-window for amplitude measurement and scoring is highlighted by the dashed vertical grey lines). (B) Horizontal topographies (top view) of the difference waves (reward minus no-reward), averaged from 235 to 285 ms, for regular (left) and special (right) trials. The black ellipse superimposed indicates $\mathrm{FCz}$ and $\mathrm{Cz}$ electrode locations. (C) Mean amplitude of the RewP for the six main conditions (regular trials), showing significant main effects of FB outcome and FB expectancy. (D) Mean amplitudes of the RewP in the $50 \%$ reward probability condition as a function of effort anticipation (absent/regular vs. present/special trials) and FB outcome (reward vs. no-reward FB). When the FB was rewarding, the RewP was larger for special compared to regular trials, without such modulation for no-reward FB, as indicated by a significant interaction between these two factors. The error bar corresponds to 1 standard error of the mean.

Figure 4. FMT results. (A) Horizontal topographies (top view) of the average FMT power change computed in the 200-400 ms window following FB onset (regular trials). When collapsing expectancy, a larger FMT power was seen for no-reward (center) than reward FB (left); (right) topography of FMT power for all conditions collapsed. (B) FMT $(4-8 \mathrm{~Hz})$ power changes from electrode $\mathrm{FCz}$, comparing reward and no-reward FB for regular vs. special trials (50\% probability condition). (C) Mean FMT power changes separately for the six main conditions (regular trials), showing significant main effects of FB expectancy and FB outcome. (D) Mean FMT power changes in the $50 \%$ reward probability condition as a function of effort anticipation and FB outcome. This analysis showed significant main effects of FB outcome and trial type, with larger FMT power values for no-reward than reward, and for special than regular trials. The error bar corresponds to 1 standard error of the mean. 
Figure 5. Delta results. (A) Horizontal topographies (top view) of the average Delta power change computed in the $200-400$ ms window following FB onset (regular trials). When collapsing expectancy, a larger centro-parietal delta power was seen for reward FB (left) than no-reward FB (center); (right) delta power for all conditions collapsed. (B) Delta (0.8-3.9 Hz) power changes from centro-parietal electrodes ( $\mathrm{CP} z, \mathrm{CP} 1, \mathrm{CP} 2, \mathrm{CP} 3$, and $\mathrm{CP} 4$ collapsed), comparing reward to no-reward FB, for regular vs. special trials (50\% probability condition). (C) Mean Delta power changes for the six main conditions (regular trials), showing significant main effects of FB expectancy and FB outcome, with larger values for unexpected than expected, and for reward than no-reward FB. (D) Mean Delta power changes in the $50 \%$ reward probability condition. Delta power was the largest when the FB was rewarding in special compared to regular trials, without such modulation for no-reward $\mathrm{FB}$, as confirmed by a trend-significant interaction between these two factors. The error bar corresponds to 1 standard error of the mean.

Figure 6. Beta-gamma results. (A) Horizontal topographies (top view) of the average Betagamma power change computed in the $200-400$ ms window following FB onset (regular trials). When collapsing expectancy, beta-gamma power increased for reward FB (left) compared to no-reward FB (center); (right) beta-gamma power for all conditions together. (B) Beta-gamma (20-35 Hz) power changes from fronto-central electrodes (FCz, FC1, FC2, and Fz collapsed), comparing reward and noreward FB, for regular vs. special trials (50\% probability condition). (C) Mean Beta-gamma power changes for the six main conditions (regular trials), showing a t main effect of FB outcome only, translating larger power values for reward than no-reward FB. (D) Mean Beta-gamma power changes in the $50 \%$ reward probability condition. A significant interaction was found between the two factors showing larger power values for reward FB in special compared to regular trials. The error bar corresponds to 1 standard error of the mean. 


\section{Supplementary Materials}

\section{Methods}

\section{Participants}

Sixteen students (14 females), recruited with the same criteria and from the same student population as the main EEG study, participated in this follow-up behavioral experiment. They were compensated with a variable 10.20-12.80€ amount for their participation.

\section{Task}

A modified version of the gambling task was devised. Trials with $25 \%$ or $75 \%$ reward probability were not included. Hence, the cue always corresponded to a $50 \%$ reward probability (i.e. pie chart half filled). Catch trials for the cue were therefore omitted because reward probability was constant throughout the experiment. Since we wanted to assess emotional feelings for the FB outcome (and compare special with regular trials), we added multiple ratings following the FB. These ratings probed the pleasantness, frustration and relief experienced following the FB, and were reported using specific visual analog scales (VAS). These three ratings were submitted each 48 times in total, $1000 \mathrm{~ms}$ after the offset of the FB. For each condition (regular vs. special trials) and outcome (reward vs. no reward), they were presented 12 times in total.

\section{Procedure}

The same procedure as in the main EEG experiment was used here, with the following changes. We used four blocks, each composed of 52 trials. In total, 104 regular and 104 special trials were presented in random order across these four blocks.

\section{Data analysis}

The VAS scores for each condition (regular vs. special trials), FB outcome (reward vs. no reward), and affective dimension (pleasantness, frustration or relief) were averaged across the 12 
presentations and across subjects. These values were then transformed to percentages setting anchors to the boundaries of the scales. One-tailed paired t-tests were run for the FB ratings probing "pleasure" and "relief", testing for higher values from reward FB in special vs. regular trials. Conversely, a onetailed paired t-test was run for the FB rating probing "frustration", testing for higher values from noreward FB in special vs. regular trials. For all analyses, significance alpha cutoff was 0.05 .

\section{Results}

Participants reported more pleasantness for the reward FB in special $(M=83.75, S E=2.31)$ compared to regular trials $(\mathrm{M}=78.27, \mathrm{SE}=2.48), t(15)=2.71, p=.008, \mathrm{~d}=0.57$. They also reported more relief for this $F B$ in special $(M=74.01, S E=3.28)$ compared to regular trials $(M=70.17, S E=3.71)$, $t(15)=1.98, p=.033, d=0.27$. By comparison, no significant difference in levels of frustration was found between the two trial types for no-reward FB $(t(15)=-0.19, p=.427)$. (Supplementary Figure 1) 


\section{Captions}

Supplementary Figure 1. Results of control behavioral experiment. Participants rated now and then, using a visual analog scale (VAS), FB (reward vs. no-reward) along three dimensions (pleasure, frustration and relief), separately for regular and special trials. Pleasure and relief were larger for reward than no-reward FB. Conversely, frustration was larger for no-reward than reward. Interestingly, when comparing special to regular trials, we found that participants reported significantly more pleasure and relief for reward FB for the former compared to the latter condition, consistent with a gain in reward processing when effort could be avoided. By comparison, no difference in negative feelings (frustration) or positive ones was found for no-reward FB between the two main conditions.

Supplementary Figure 2. Results of cue-locked analyses. (A) Horizontal topographies of the average FMT power during the cue presentation $(600 \mathrm{~ms})$ separately for regular and special trials. In the pre-cue interval, these two conditions were not properly matched because a previous additional cue, informing about the onset of a special case, was displayed for special trials only. This additional event in special trials created a burst in the Theta activity that lasted until the pre-cue interval. To overcome this problem, we extracted the FMT power along the whole cue epoch by means of Fast Fourier Transform and applied a topographical normalization. FMT power at each site was divided by the summed FMT power across all sites, and a within-subject Z-score was then obtained by normalizing over all electrodes. A paired samples T-test performed on normalized Theta power, extracted from channel FCz, showed an increased FMT activity for special compared to regular trials $[\mathrm{t}(25)=2.863, \mathrm{p}$ $=0.008, d=0.562$ ]. (B) Grand average ERP waveforms (channel $\mathrm{Cz}$ ) time-locked to the cue, separately for regular and special trials. The shadowing indicates one standard error of the mean. The CNV (contingent negativity variation) was defined as the mean ERP activity at electrode Cz extracted during the $1000-2000 \mathrm{~ms}$ interval following cue onset, relative to a $250 \mathrm{~ms}$ pre-cue baseline interval. Its amplitude was similar between these two conditions $[t(25)=1.012, p=0.321]$. 
Table 1. Data from the six individual pilot participants (none of them participated to the main EEG experiment or control behavioral experiment) tested prior to the EEG experiment to specify the optimal number of clicks to be used (8) for the random dot clicking task, as well as reward magnitude for the following (redo) gamble (10 eurocent) (special trials). In these conditions ( 8 clicks and 10 eurocent), we found that participants were inclined to redo the gamble most of the time, which was an important pre-requisite for our EEG data analysis and the comparison of reward processing at the FB level (following the first gamble) when effort anticipation was absent vs. present (and later exerted). 


\section{References}

Admon, R., Pizzagalli, D.A., 2015. Dysfunctional reward processing in depression. Curr. Opin. Psychol. 4, 114-118. doi:10.1016/j.copsyc.2014.12.011

Apps, M.A.J., Grima, L.L., Manohar, S., Husain, M., 2015. The role of cognitive effort in subjective reward devaluation and risky decision-making. Sci. Rep. 5, 1-11. doi:10.1038/srep16880

Arbel, Y., Goforth, K., Donchin, E., 2013. The Good, the Bad, or the Useful? The Examination of the Relationship between the Feedback-related Negativity (FRN) and Long-term Learning Outcomes. J. Cogn. Neurosci. 25, 1249-1260. doi:10.1162/jocn_a_00385

Aston-Jones, G., Cohen, J.D., 2005. AN INTEGRATIVE THEORY OF LOCUS COERULEUSNOREPINEPHRINE FUNCTION: Adaptive Gain and Optimal Performance. Annu. Rev. Neurosci. 28, 403-450. doi:10.1146/annurev.neuro.28.061604.135709

Baker, T.E., Holroyd, C.B., 2011. Dissociated roles of the anterior cingulate cortex in reward and conflict processing as revealed by the feedback error-related negativity and N200. Biol. Psychol. 87, 25-34. doi:10.1016/j.biopsycho.2011.01.010

Bernat, E.M., Nelson, L.D., Baskin-Sommers, A.R., 2015. Time-Frequency Theta and Delta Measures Index Separable Components of Feedback Processing in a Gambling Task 33, 395-401. doi:10.1038/nbt.3121.ChIP-nexus

Berridge, K.C., Robinson, T.E., 2003. Parsing reward. Trends Neurosci. 26, 507-513. doi:10.1016/S0166-2236(03)00233-9

Berridge, K.C., Robinson, T.E., 1998. What is the role of dopamine in reward: Hedonics, learning, or incentive salience? Brain Res. Rev. 28, 308-67. doi:10.1016/\$0165-0173(98)00019-8

Berridge, K.C., Robinson, T.E., Aldridge, J.W., 2010. Dissectin components of reward: "liking", 
"wanting", and learning 9, 65-73. doi:10.1016/j.coph.2008.12.014.Dissecting

Botvinick, M., Braver, T., 2015. Motivation and Cognitive Control: From Behavior to Neural Mechanism. Annu. Rev. Psychol. 66, 83-113. doi:10.1146/annurev-psych-010814-015044

Cavanagh, J.F., Figueroa, C.M., Cohen, M.X., Frank, M.J., 2012. Frontal theta reflects uncertainty and unexpectedness during exploration and exploitation. Cereb. Cortex 22, 2575-2586. doi:10.1093/cercor/bhr332

Cavanagh, J.F., Frank, M.J., 2014. Frontal Theta as a Mechanism for cognitive control. Psychophysiology 51, S8-S8. doi:10.1016/j.tics.2014.04.012.Frontal

Cavanagh, J.F., Frank, M.J., Klein, T.J., Allen, J.J.B., 2010. Frontal theta links prediction errors to behavioral adaptation in reinforcement learning. Neuroimage 49, 3198-3209. doi:10.1016/j.neuroimage.2009.11.080

Cavanagh, J.F., Shackman, A.J., 2015. Frontal midline theta reflects anxiety and cognitive control: Meta-analytic evidence. J. Physiol. Paris 109, 3-15. doi:10.1016/j.jphysparis.2014.04.003

Charnov, E.L., 1976. Optimal foraging, the marginal value theorem. Theor. Popul. Biol. 9, 129-136. doi:10.1016/0040-5809(76)90040-X

Chase, H.W., Swainson, R., Durham, L., Benham, L., Cools, R., 2011. Feedback-related Negativity Codes Prediction Error but Not Behavioral Adjustment during Probabilistic Reversal Learning. J. Cogn. Neurosci. 23, 936-946. doi:10.1162/jocn.2010.21456

Chong, T.T.J., Apps, M., Giehl, K., Sillence, A., Grima, L.L., Husain, M., 2017. Neurocomputational mechanisms underlying subjective valuation of effort costs. PLoS Biol. 15, 1-28. doi:10.1371/journal.pbio.1002598

Cohen, M.X., 2014. Analyzing neural time series data: theory and practice. MIT Press.

Cohen, M.X., Donner, T.H., 2013. Midfrontal conflict-related theta-band power reflects neural 
oscillations that predict behavior. J. Neurophysiol. 110, 2752-2763. doi:10.1152/jn.00479.2013

Cohen, M.X., Elger, C.E., Ranganath, C., 2007. Reward expectation modulates feedback-related negativity and EEG spectra. Neuroimage 35, 968-978. doi:10.1016/j.neuroimage.2006.11.056

Croxson, P.L., Walton, M.E., Reilly, J.X.O., Behrens, T.E.J., Rushworth, M.F.S., 2009. Effort-Based Cost - Benefit Valuation and the Human Brain 29, 4531-4541. doi:10.1523/JNEUROSCI.4515-08.2009

De Bruijn, E.R.A., Hulstijn, W., Verkes, R.J., Ruigt, G.S.F., Sabbe, B.G.C., 2004. Drug-induced stimulation and suppression of action monitoring in healthy volunteers. Psychopharmacology (Berl). 177, 151-160. doi:10.1007/s00213-004-1915-6

Delorme, A., Makeig, S., 2004. EEGLAB: an open source toolbox for analysis of single-trial EEG dynamics including independent component analysis. J. Neurosci. Methods 134, 9-21. doi:10.1016/j.jneumeth.2003.10.009

Eppinger, B., Kray, J., Mock, B., Mecklinger, A., 2008. Better or worse than expected? Aging, learning, and the ERN. Neuropsychologia 46, 521-539. doi:10.1016/j.neuropsychologia.2007.09.001

Foti, D., Weinberg, A., Dien, J., Hajcak, G., 2011. Event-related potential activity in the basal ganglia differentiates rewards from nonrewards: Response to commentary. Hum. Brain Mapp. 32, 2267-2269. doi:10.1002/hbm.21357

Gheza, D., Paul, K., Pourtois, G., 2017. Dissociable effects of reward and expectancy during evaluative feedback processing revealed by topographic ERP mapping analysis. Int. J. Psychophysiol. doi:10.1016/J.IJPSYCHO.2017.11.013

Hajcak, G., Holroyd, C.B., Moser, J.S., Simons, R.F., 2005. Brain potentials associated with expected and unexpected good and bad outcomes. Psychophysiology 42, 161-170. doi:10.1111/j.14698986.2005.00278.x

Hajcak, G., Moser, J.S., Holroyd, C.B., Simons, R.F., 2007. It's worse than you thought: The feedback 
negativity and violations of reward prediction in gambling tasks. Psychophysiology 44, 905-912. doi:10.1111/j.1469-8986.2007.00567.x

Hajihosseini, A., Holroyd, C.B., 2013. Frontal midline theta and N200 amplitude reflect complementary information about expectancy and outcome evaluation. Psychophysiology 50, 550-562. doi:10.1111/psyp.12040

HajiHosseini, A., Rodr??guez-Fornells, A., Marco-Pallar??s, J., 2012. The role of beta-gamma oscillations in unexpected rewards processing. Neuroimage 60, 1678-1685. doi:10.1016/j.neuroimage.2012.01.125

Holroyd, C.B., Coles, M.G.H., 2002. The neural basis of human error processing: Reinforcement learning, dopamine, and the error-related negativity. Psychol. Rev. 109, 679-709. doi:10.1037/0033-295X.109.4.679

Holroyd, C.B., McClure, S.M., 2015. Hierarchical control over effortful behavior by rodent medial frontal cortex: A computational model. Psychol. Rev. 122, 54-83. doi:10.1037/a0038339

Holroyd, C.B., Pakzad-Vaezi, K.L., Krigolson, O.E., 2008. The feedback correct-related positivity: Sensitivity of the event-related brain potential to unexpected positive feedback. Psychophysiology 45, 688-697. doi:10.1111/j.1469-8986.2008.00668.x

Holroyd, C.B., Umemoto, A., 2016. The research domain criteria framework: The case for anterior cingulate cortex. Neurosci. Biobehav. Rev. 71, 418-443. doi:10.1016/j.neubiorev.2016.09.021 Holroyd, C.B., Yeung, N., 2012. Motivation of extended behaviors by anterior cingulate cortex. Trends Cogn. Sci. 16, 122-128. doi:10.1016/j.tics.2011.12.008

Hull, C., 1943. Principles of Behavior. Appleton-Century-Crofts, New York, USA. JASP Team, T., 2017. JASP (Version 0.8.2)[Computer software]. Jocham, G., Ullsperger, M., 2009. Neuropharmacology of performance monitoring. Neurosci. 
Biobehav. Rev. doi:10.1016/j.neubiorev.2008.08.011

Keil, A., Debener, S., Gratton, G., Junghöfer, M., Kappenman, E.S., Luck, S.J., Luu, P., Miller, G.A., Yee, C.M., 2014. Committee report: Publication guidelines and recommendations for studies using electroencephalography and magnetoencephalography. Psychophysiology 51, 1-21. doi:10.1111/psyp.12147

Klein, E.D., Bhatt, R.S., Zentall, T.R., 2005. Contrast and the justification of effort. Psychon. Bull. Rev. 12, 335-339. doi:10.3758/BF03196381

Kool, W., McGuire, J.T., Rosen, Z.B., Botvinick, M.M., 2010. Decision Making and the Avoidance of Cogntive Demand. J. Exp. Psychol. Gen. 139, 665-682. doi:10.1037/a0020198.Decision

Kurniawan, I.T., Guitart-Masip, M., Dayan, P., Dolan, R.J., 2013. Effort and Valuation in the Brain: The Effects of Anticipation and Execution. J. Neurosci. 33, 6160-6169. doi:10.1523/JNEUROSCI.4777-12.2013

Kurniawan, I.T., Seymour, B., Talmi, D., Yoshida, W., Chater, N., Dolan, R.J., 2010. Choosing to make an effort: the role of striatum in signaling physical effort of a chosen action. J. Neurophysiol. 104, 313-21. doi:10.1152/jn.00027.2010

Kurzban, R., Duckworth, A., Kable, J.W., Myers, J., 2013. An opportunity cost model of subjective effort and task performance. Behav. Brain Sci. 36, 661-679. doi:10.1017/S0140525X12003196

Luck, S.J., Gaspelin, N., 2017. How to get statistically significant effects in any ERP experiment (and why you shouldn't). Psychophysiology 54, 146-157. doi:10.1111/psyp.12639

Ma, L., Hyman, J.M., Phillips, A.G., Seamans, J.K., 2014. Tracking Progress toward a Goal in Corticostriatal Ensembles. J. Neurosci. 34, 2244-2253. doi:10.1523/JNEUROSCI.3834-13.2014

Marco-Pallares, J., Cucurell, D., Cunillera, T., Garc??a, R., Andr??s-Pueyo, A., M??nte, T.F., Rodr??guez-Fornells, A., 2008. Human oscillatory activity associated to reward processing in a 
gambling task. Neuropsychologia 46, 241-248. doi:10.1016/j.neuropsychologia.2007.07.016

Marco-Pallares, J., Cucurell, D., Münte, T.F., Strien, N., Rodriguez-Fornells, A., 2011. On the number of trials needed for a stable feedback-related negativity. Psychophysiology 48, 852-860. doi:10.1111/j.1469-8986.2010.01152.x

Mas-Herrero, E., Ripollés, P., HajiHosseini, A., Rodríguez-Fornells, A., Marco-Pallarés, J., 2015. Beta oscillations and reward processing: Coupling oscillatory activity and hemodynamic responses. Neuroimage 119, 13-19. doi:10.1016/j.neuroimage.2015.05.095

Mussel, P., Ulrich, N., Allen, J.J.B., Osinsky, R., Hewig, J., 2016. Patterns of theta oscillation reflect the neural basis of individual differences in epistemic motivation. Sci. Rep. 6, 29245. doi:10.1038/srep29245

Novak, K.D., Foti, D., 2015. Teasing apart the anticipatory and consummatory processing of monetary incentives: An event-related potential study of reward dynamics. Psychophysiology 52, 14701482. doi:10.1111/psyp.12504

Olbrich, S., Arns, M., 2013. EEG biomarkers in major depressive disorder: Discriminative power and prediction of treatment response. Int. Rev. Psychiatry 25, 604-618. doi:10.3109/09540261.2013.816269

Oliveira, F.T.P., McDonald, J.J., Goodman, D., 2007. Performance Monitoring in the Anterior Cingulate is Not All Error Related: Expectancy Deviation and the Representation of Action-Outcome Associations. J. Cogn. Neurosci. 19, 1994-2004. doi:10.1162/jocn.2007.19.12.1994

Paul, K., Pourtois, G., 2017. Mood congruent tuning of reward expectation in positive mood: evidence from FRN and theta modulations. Soc. Cogn. Affect. Neurosci. 1-10. doi:10.1093/scan/nsx010

Pessiglione, M., Schmidt, L., Draganski, B., Kalisch, R., Lau, H., Dolan, R.J., Frith, C.D., 2007. How the Brain Translates Money into Force: A Neuroimaging Study of Subliminal Motivation 316, 904- 
907.

Pizzagalli, D.A., 2014. Depression, Stress, and Anhedonia: Toward a Synthesis and Integrated Model. Annu. Rev. Clin. Psychol. 10, 393-423. doi:10.1146/annurev-clinpsy-050212-185606

Potts, G.F., Martin, L.E., Burton, P., Montague, P.R., 2006. When things are better or worse than expected: the medial frontal cortex and the allocation of processing resources. J. Cogn. Neurosci. 18, 1112-1119. doi:10.1162/jocn.2006.18.7.1112

Prévost, C., Pessiglione, M., Métérau, E., Cléry-Melin, M.-L., Dreher, J.-C., 2010. Separate Valuation Subsystems for Delay and Effort Decision 30, 14080-14090. doi:10.1523/JNEUROSCI.275210.2010

Proudfit, G.H., 2015. The reward positivity: From basic research on reward to a biomarker for depression. Psychophysiology 52, 449-459. doi:10.1111/psyp.12370

Proudfit, G.H., Bress, J.N., Foti, D., Kujawa, A., Klein, D.N., 2015. Depression and event-related potentials: Emotional disengagement and reward insensitivity. Curr. Opin. Psychol. 4, 110-113. doi:10.1016/j.copsyc.2014.12.018

Riba, J., Rodríguez-Fornells, A., Morte, A., Münte, T.F., Barbanoj, M.J., 2005. Noradrenergic stimulation enhances human action monitoring. J. Neurosci. 25, 4370-4. doi:10.1523/JNEUROSCI.4437-04.2005

Rouder, J.N., Morey, R.D., Verhagen, J., Swagman, A.R., Wagenmakers, E.-J., 2017. Bayesian analysis of factorial designs. Psychol. Methods 22, 304-321. doi:10.1037/met0000057

Salamone, J.D., Correa, M., 2012. The Mysterious Motivational Functions of Mesolimbic Dopamine. Neuron 76, 470-485. doi:10.1016/j.neuron.2012.10.021

Salamone, J.D., Correa, M., Farrar, A., Mingote, S.M., 2007. Effort-related functions of nucleus accumbens dopamine and associated forebrain circuits. Psychopharmacology (Berl). 191, 461- 
482. doi:10.1007/s00213-006-0668-9

Salamone, J.D., Correa, M., Mingote, S., Weber, S.M., 2003. Nucleus Accumbens Dopamine and the Regulation of Effort in Food-Seeking Behavior: Implications for Studies of Natural Motivation, Psychiatry, and Drug Abuse. J. Pharmacol. Exp. Ther. 305, 1-8. doi:10.1124/jpet.102.035063

Salamone, J.D., Correa, M., Nunes, E.J., Randall, P.A., Pardo, M., 2012. The Behavioral Pharmacology of Effort-related Choice Behavior: Dopamine, Adenosine and Beyond. J. Exp. Anal. Behav. 97, 125-146. doi:10.1901/jeab.2012.97-125

Sallet, J., Camille, N., Procyk, E., 2013. Modulation of feedback-related negativity during trial-anderror exploration and encoding of behavioral shifts. Front. Neurosci. 7, 1-10. doi:10.3389/fnins.2013.00209

Sambrook, T.D., Goslin, J., 2014. Mediofrontal event-related potentials in response to positive, negative and unsigned prediction errors. Neuropsychologia 61, 1-10. doi:10.1016/j.neuropsychologia.2014.06.004

Sherdell, L., Waugh, C.E., Gotlib, I.H., 2012. Anticipatory Pleasure Predicts Motivation for Reward in Major Depression 121, 51-60. doi:10.1037/a0024945

Smith, X.E.H., Banks, X.G.P., Mikell, C.B., Cash, X.S.S., Patel, S.R., Eskandar, E.N., Sheth, S.A., 2015. Frequency-Dependent Representation of Reinforcement- Related Information in the Human Medial and Lateral Prefrontal Cortex 35, 15827-15836. doi:10.1523/JNEUROSCI.1864-15.2015

Treadway, M.T., Bossaller, N., Shelton, R.C., Zald, D.H., 2012. Effort-Based Decision-Making in Major Depressive Disorder: a Translational Model of Motivational Anhedonia 121, 553-558. doi:10.1037/a0028813.Effort-Based

Treadway, M.T., Buckholtz, J.W., Schwartzman, A.N., Lambert, W.E., Zald, D.H., 2009. Worth the "EEfRT"? The effort expenditure for rewards task as an objective measure of motivation and anhedonia. PLoS One 4, 1-9. doi:10.1371/journal.pone.0006598 
Ullsperger, M., 2017. Neural Bases of Performance Monitoring.

Ullsperger, M., Danielmeier, C., Jocham, G., 2014a. Neurophysiology of Performance Monitoring and Adaptive Behavior. Physiol. Rev. 94, 35-79. doi:10.1152/physrev.00041.2012

Ullsperger, M., Fischer, A.G., Nigbur, R., Endrass, T., 2014b. Neural mechanisms and temporal dynamics of performance monitoring. Trends Cogn. Sci. 18, 259-267. doi:10.1016/j.tics.2014.02.009

Vassena, E., Holroyd, C.B., Alexander, W.H., 2017. Computational models of anterior cingulate cortex: At the crossroads between prediction and effort. Front. Neurosci. 11, 1-9. doi:10.3389/fnins.2017.00316

Vassena, E., Silvetti, M., Boehler, C.N., Achten, E., Fias, W., Verguts, T., 2014. Overlapping neural systems represent cognitive effort and reward anticipation. PLoS One 9. doi:10.1371/journal.pone.0091008

Verguts, T., Vassena, E., Silvetti, M., 2015. Adaptive effort investment in cognitive and physical tasks: a neurocomputational model. Front. Behav. Neurosci. 9. doi:10.3389/fnbeh.2015.00057

Walsh, M.M., Anderson, J.R., 2012. Learning from experience: Event-related potential correlates of reward processing, neural adaptation, and behavioral choice. Neurosci. Biobehav. Rev. 36, 1870-1884. doi:10.1016/j.neubiorev.2012.05.008

Wascher, E., Rasch, B., S??nger, J., Hoffmann, S., Schneider, D., Rinkenauer, G., Heuer, H., Gutberlet, I., 2014. Frontal theta activity reflects distinct aspects of mental fatigue. Biol. Psychol. 96, 5765. doi:10.1016/j.biopsycho.2013.11.010

Webb, C.A., Auerbach, R.P., Bondy, E., Stanton, C.H., Foti, D., Pizzagalli, D.A., 2017. Abnormal neural responses to feedback in depressed adolescents. J. Abnorm. Psychol. 126, 19-31. doi:10.1037/abn0000228 
Weinberg, A., Riesel, A., Proudfit, G.H., 2014. Show me the Money: The impact of actual rewards and losses on the feedback negativity. Brain Cogn. 87, 134-139. doi:10.1016/j.bandc.2014.03.015

Weinberg, A., Shankman, S.A., 2017. Blunted Reward Processing in Remitted Melancholic Depression. Clin. Psychol. Sci. 5, 14-25. doi:10.1177/2167702616633158

Westbrook, A., Braver, T.S., 2016. Dopamine Does Double Duty in Motivating Cognitive Effort. Neuron 89, 695-710. doi:doi: 10.1016/j.neuron.2015.12.029.

Westbrook, A., Braver, T.S., 2015. Cognitive effort: A neuroeconomic approach. Cogn. Affect. Behav. Neurosci. 15, 395-415. doi:10.3758/s13415-015-0334-y

Whitton, A.E., Kakani, P., Foti, D., Van'T Veer, A., Haile, A., Crowley, D.J., Pizzagalli, D.A., 2016. Blunted Neural Responses to Reward in Remitted Major Depression: A High-Density EventRelated Potential Study. Biol. Psychiatry Cogn. Neurosci. Neuroimaging 1, 87-95. doi:10.1016/j.bpsc.2015.09.007

Yeung, N., Sanfey, A.G., 2004. Independent Coding of Reward Magnitude and Valence in the Human Brain. J. Neurosci. 24, 6258-6264. doi:10.1523/JNEUROSCI.4537-03.2004 


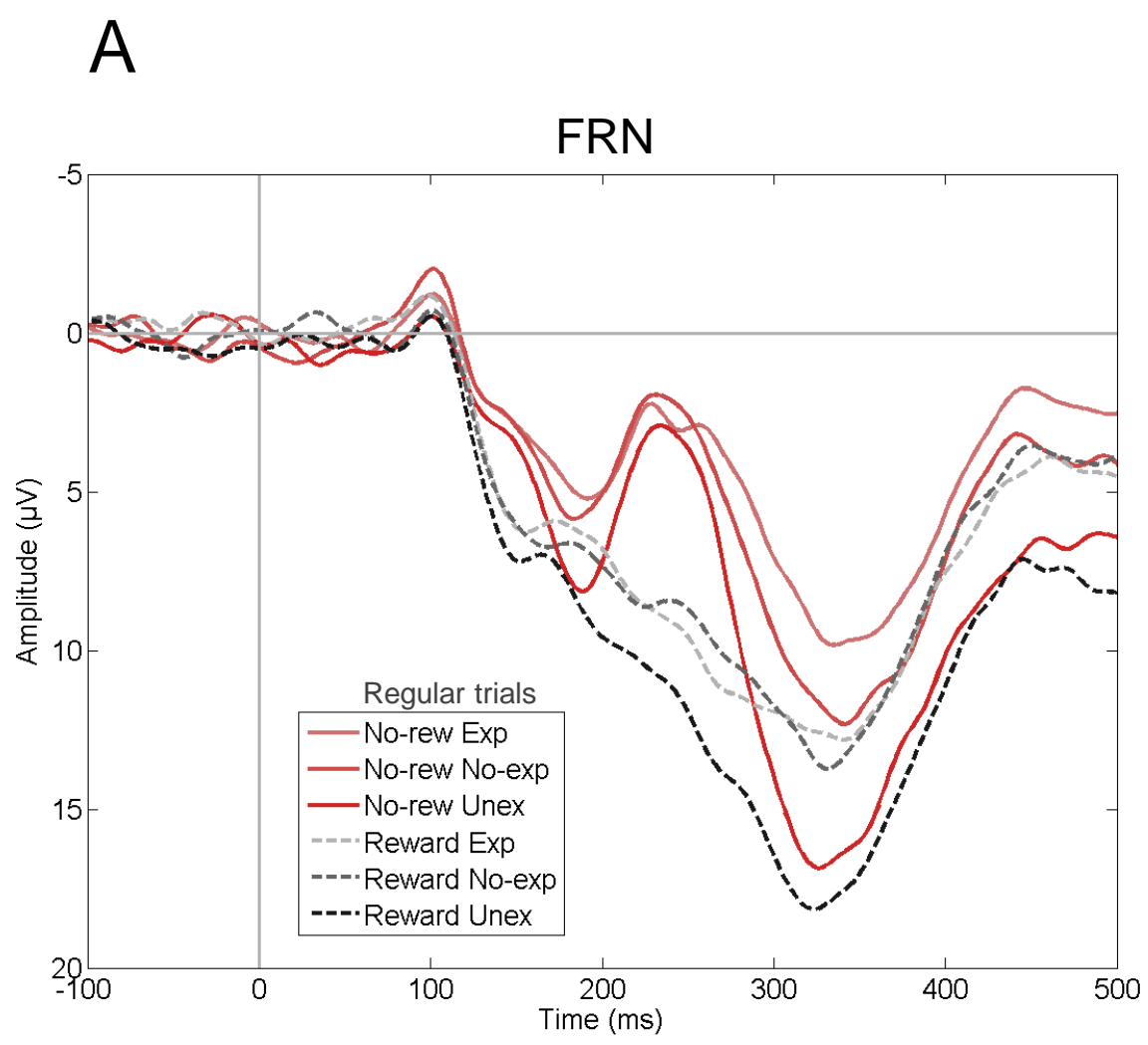

B

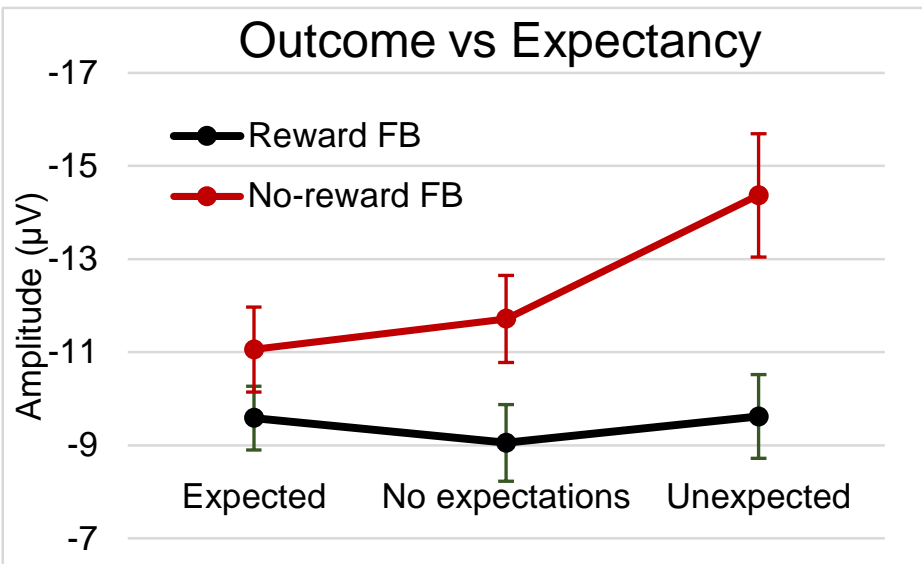

C

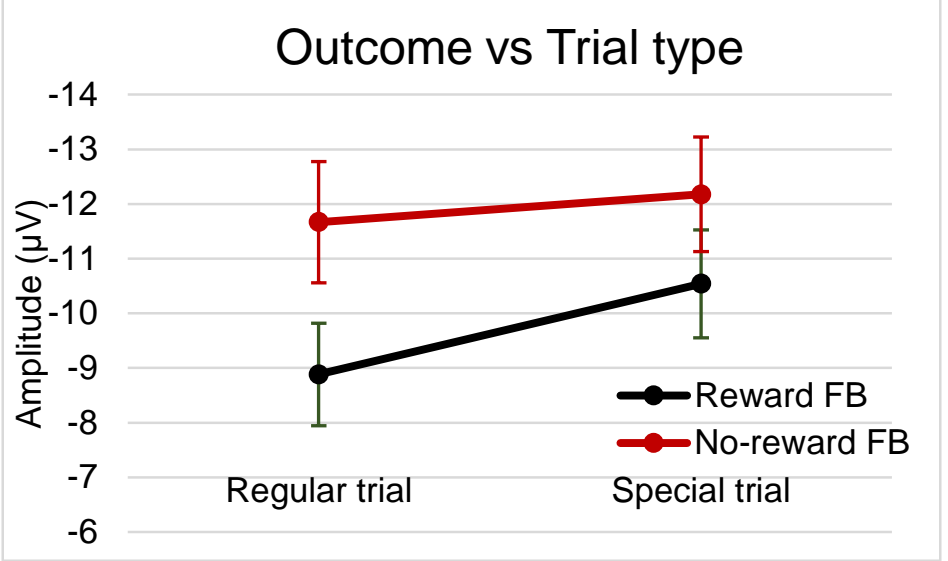

FIGURE 2 


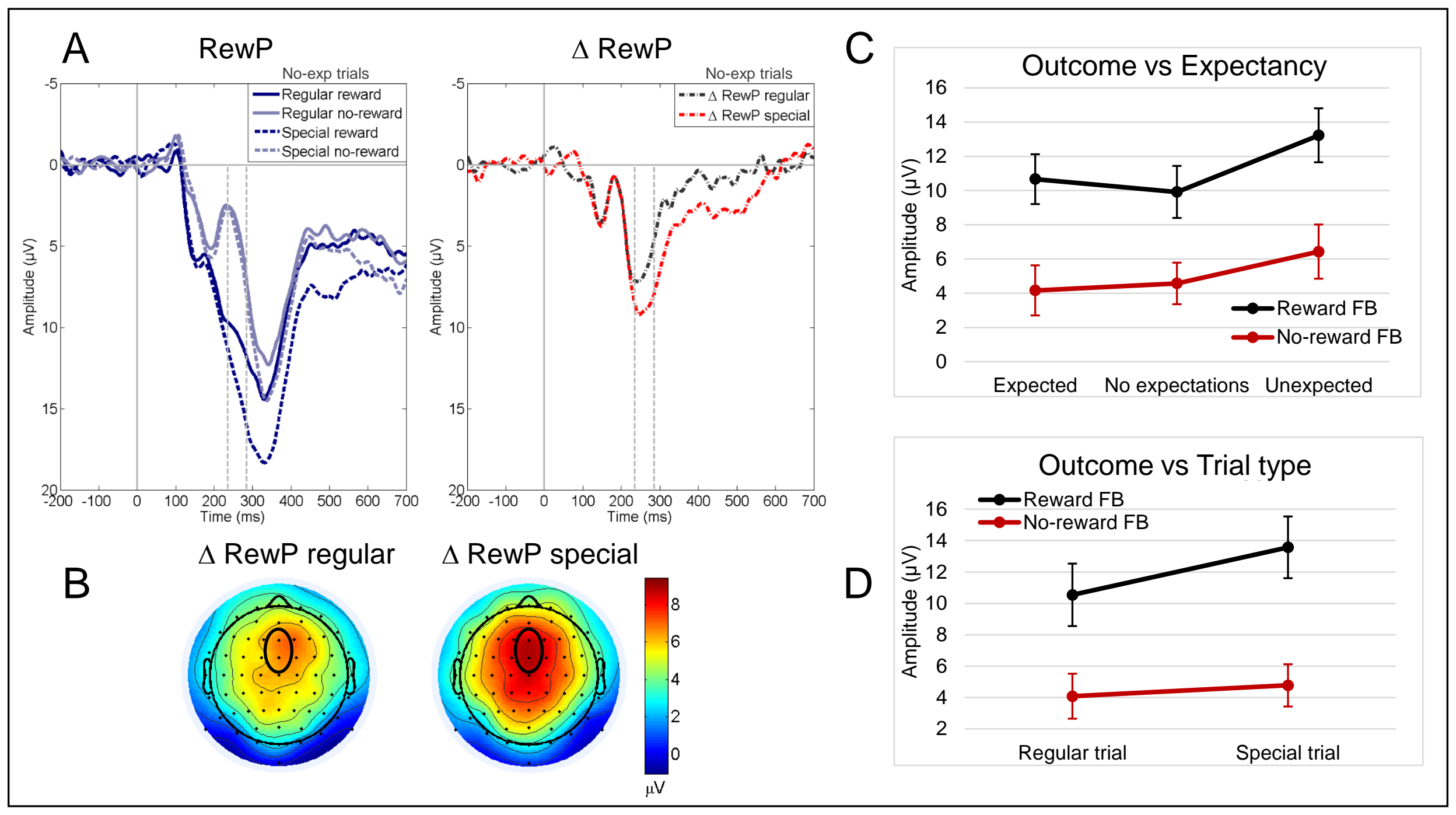

FIGURE 3 
Frontal midline Theta power

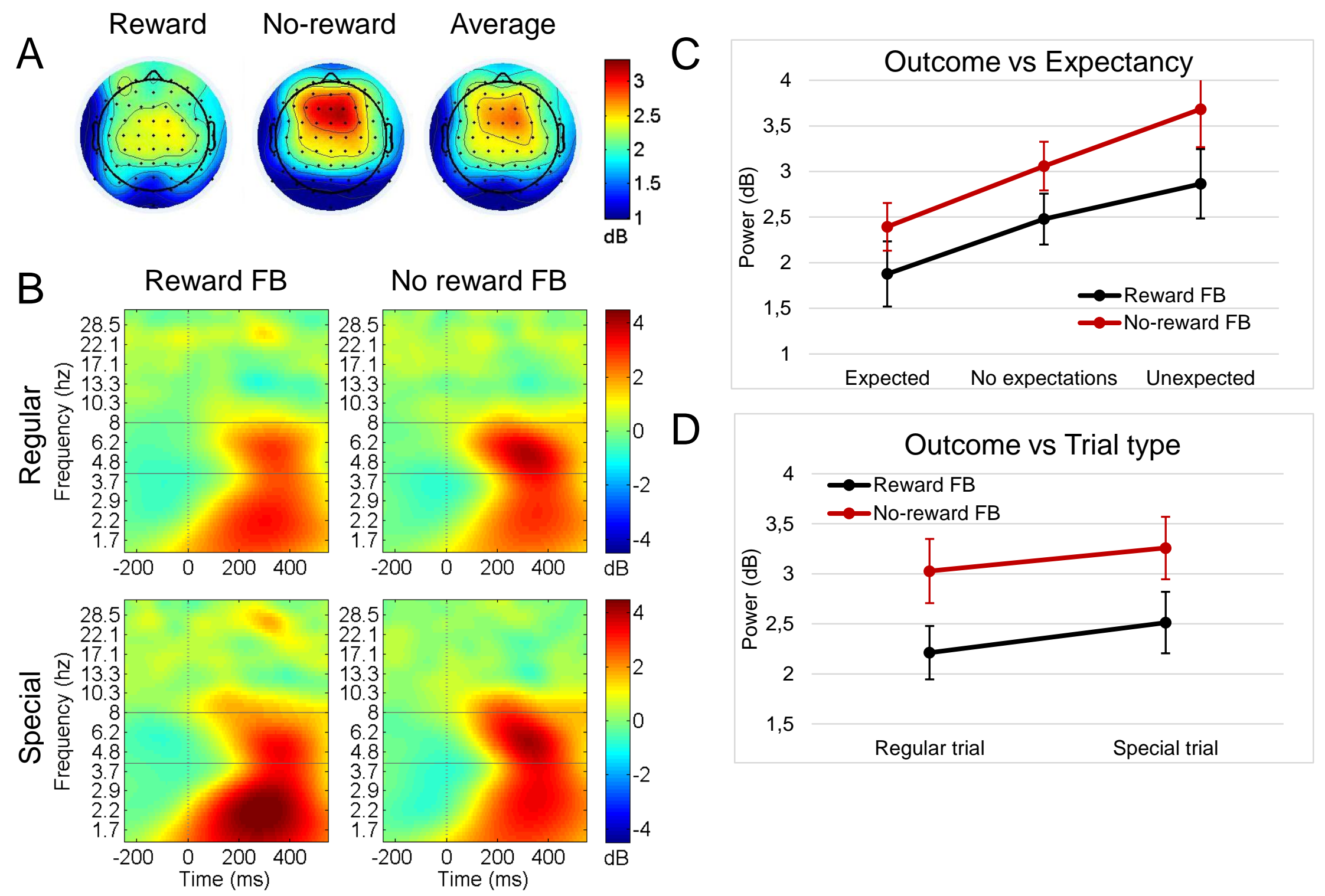




\section{Delta power}
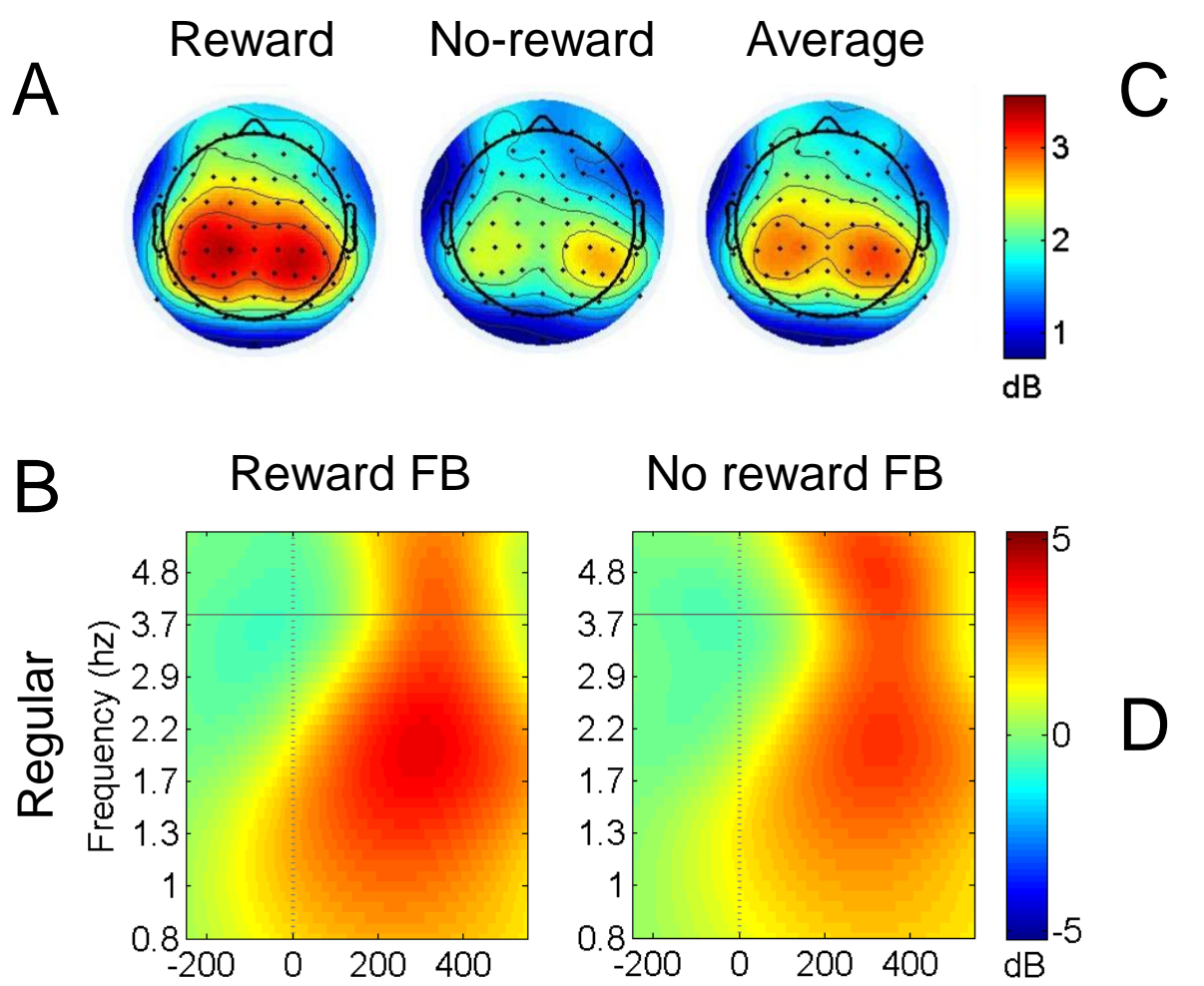

No reward FB
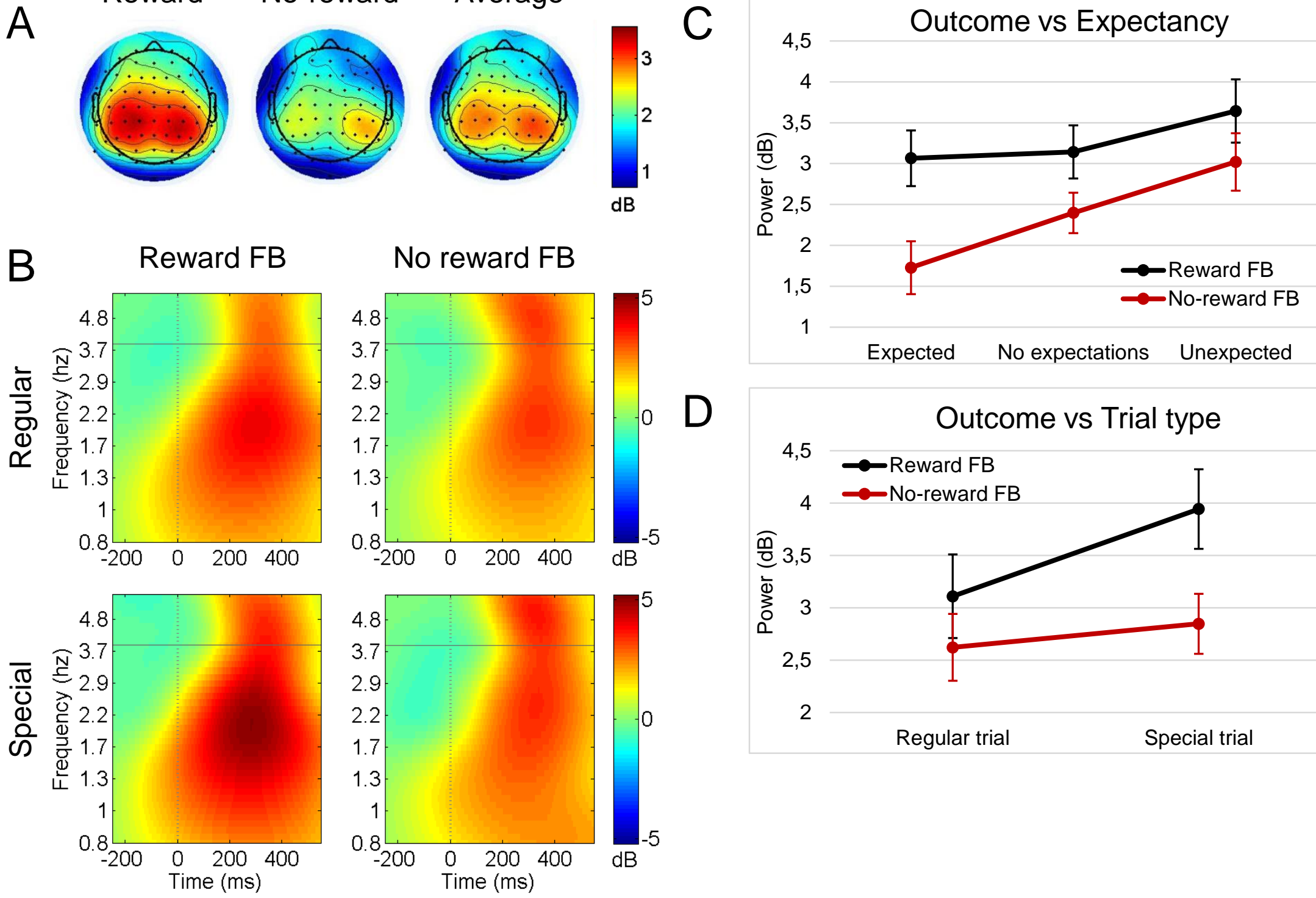

D

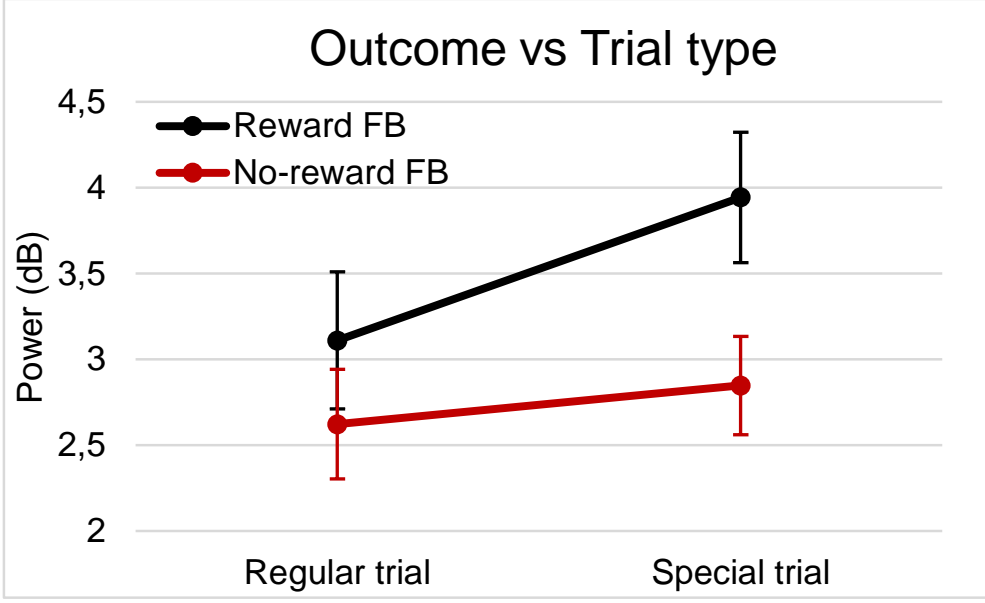

FIGURE 5 
Beta-gamma power

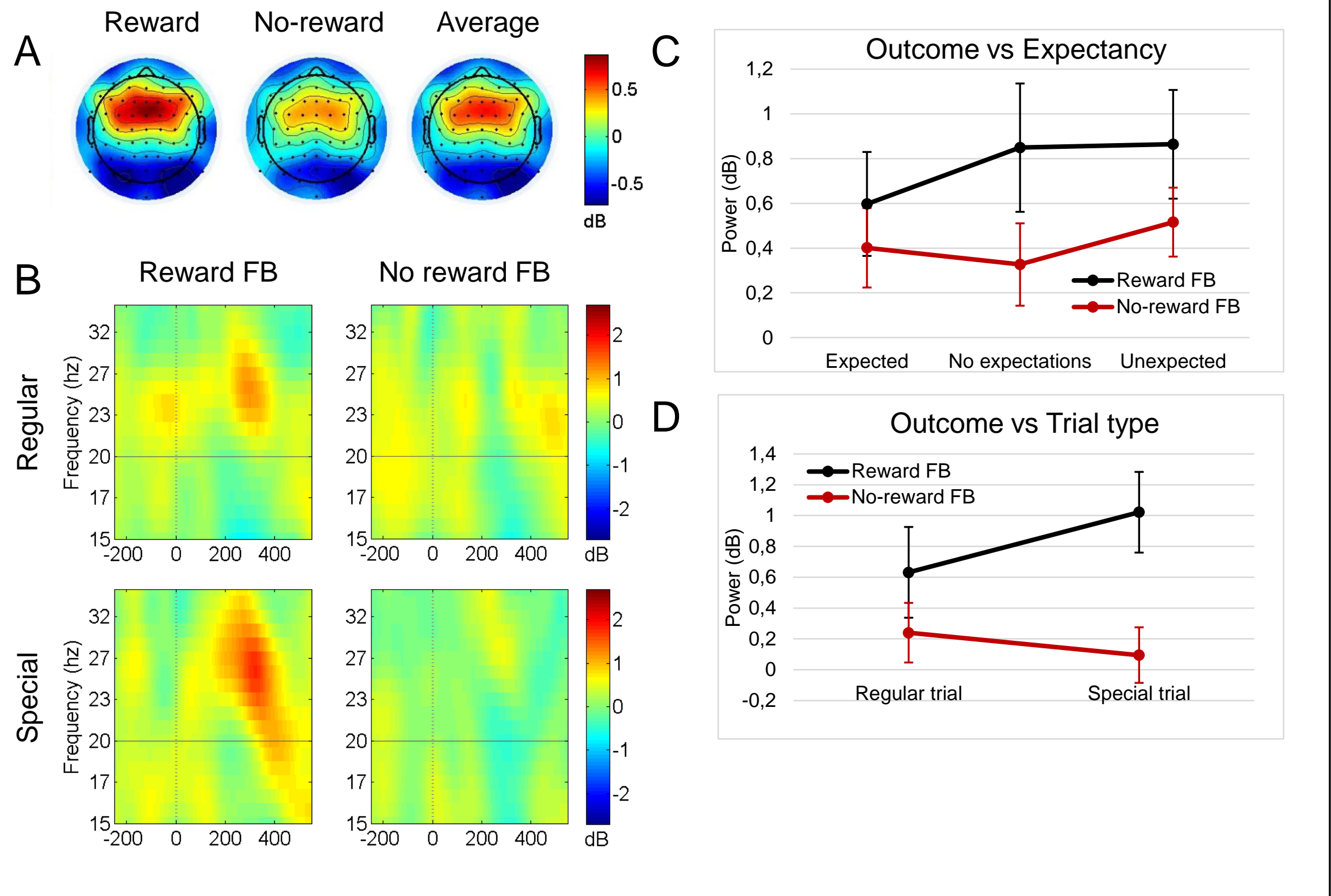

FIGURE 6 


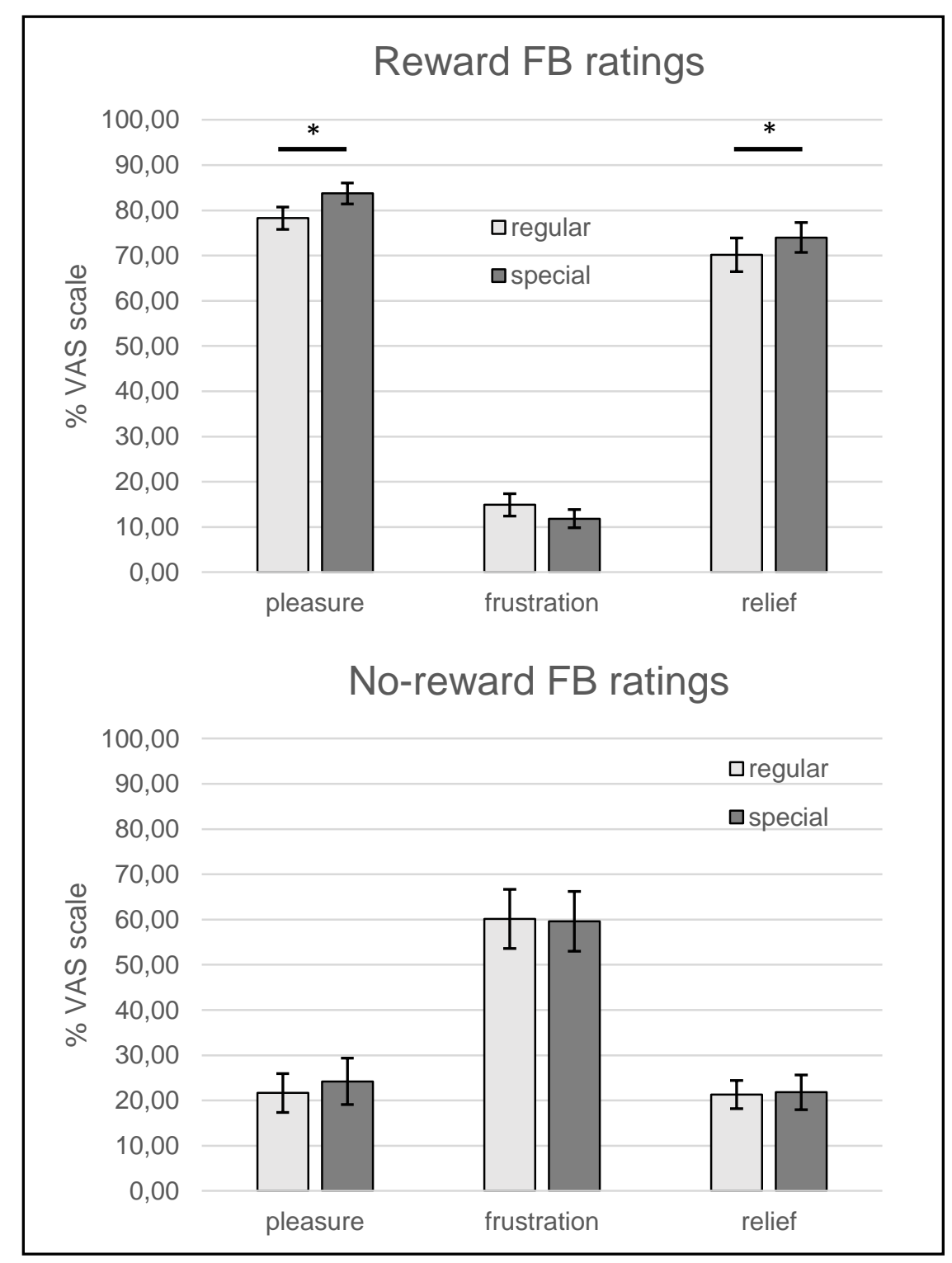

Supplementary Figure 1 
A
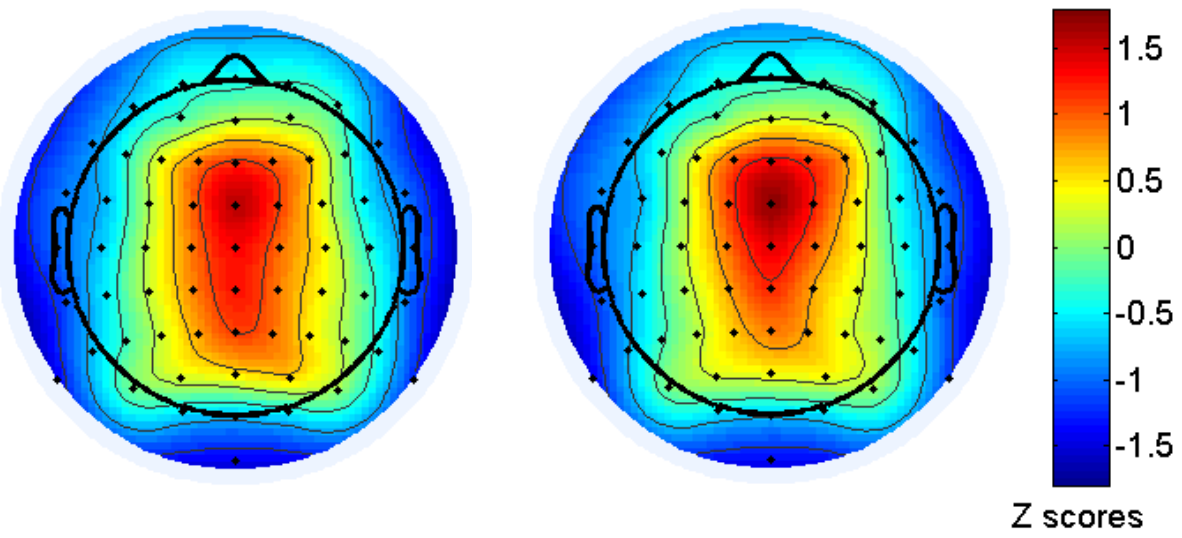

B

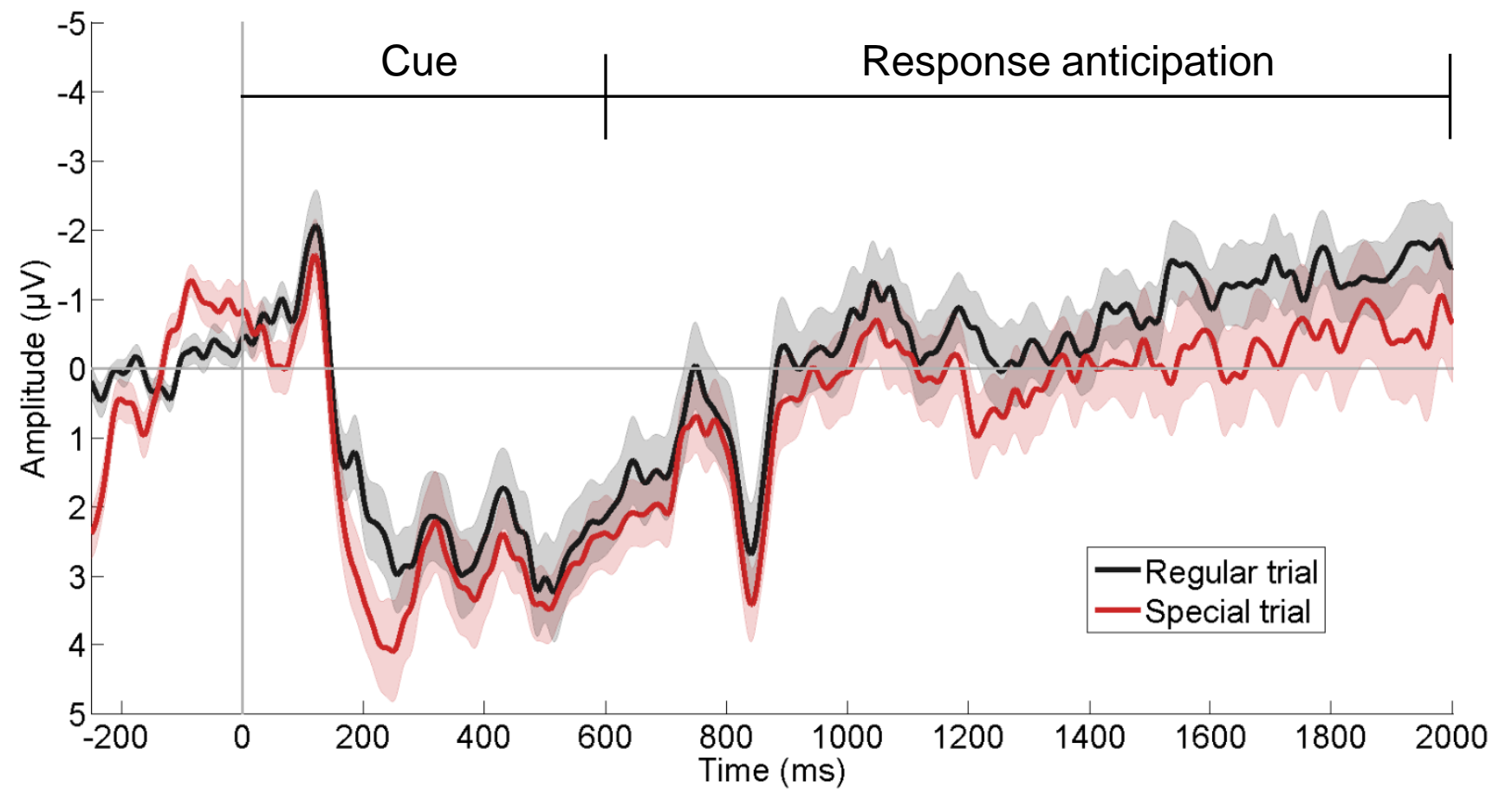

Supplementary Figure 2 
Supplementary Table 1

Data from the six pilot participants

\begin{tabular}{cccc}
\hline pilot & clicks & reward & $\%$ yes \\
\hline 1 & 5 & 10 & 96 \\
2 & 8 & 10 & 63 \\
3 & 20 & 10 & 0 \\
4 & 10 & 5 & 7 \\
5 & 8 & 10 & 90 \\
6 & 8 & 10 & 17 \\
\hline
\end{tabular}

